\title{
Convergence of iterative sequences for fixed points of an infinite family of nonexpansive mappings based on a hybrid steepest descent methods
}

Nawitcha Onjai-uea ${ }^{1,2}$, Chaichana Jaiboon ${ }^{2,3^{*}}$, Poom Kumam ${ }^{1,2}$ and Usa Wannasingha Humphries ${ }^{1,2}$

* Correspondence: chaichana. j@rmutr.ac.th

${ }^{2}$ Centre of Excellence in Mathematics, CHE, Si Ayuthaya Road, Bangkok 10400, Thailand Full list of author information is available at the end of the article

\begin{abstract}
The propose of this article is to consider the strong convergence of an iterative sequences for finding a common element of the set of fixed points of an infinite family of nonexpansive mappings, the set of solutions of the variational inequalities for inverse strongly monotone mappings, and the set of solutions of system of equilibrium problems in Hilbert spaces by using a hybrid steepest descent methods. Our results improve and generalize many known corresponding results.

AMS (2000) Subject Classification: 46C05; 47H09; 47H10.

Keywords: nonexpansive mappings, system equilibrium problem, variational inequality problem, hybrid steepest descent method
\end{abstract}

\section{Introduction}

Let $H$ be a real Hilbert space whose inner product and norm are denoted by $\langle\cdot, \cdot\rangle$ and $\|\cdot\|$, respectively. Let $C$ be a nonempty closed convex subset of $H$ and let $F: C \times C \rightarrow$ $\mathbb{R}$ be a bifunction, where $\mathbb{R}$ is the set of real numbers. The equilibrium problem for $F$ : $C \times C \rightarrow \mathbb{R}$ is to find $x^{*} \in C$ such that

$$
F\left(x^{*}, y\right) \geq 0, \quad \forall y \in C .
$$

The set of solutions of (1.1) is denoted by $E P(F)$.

Let $\left\{F_{i}, i=1,2, \ldots, N\right\}$ be a finite family of bifunctions from $C \times C$ into $\mathbb{R}$, where $\mathbb{R}$ is the set of real numbers. The system of equilibrium problems for $\left\{F_{1}, F_{2}, \ldots, F_{N}\right\}$ is to find a common element $x^{*} \in C$ such that

$$
\begin{cases}F_{1}\left(x^{*}, y\right) \geq 0, & \forall y \in C, \\ F_{2}\left(x^{*}, y\right) \geq 0, & \forall y \in C, \\ \vdots & \\ F_{N}\left(x^{*}, y\right) \geq 0, & \forall y \in C .\end{cases}
$$

We denote the set of solutions of (1.2) by $\cap_{i=1}^{N} \operatorname{SEP}\left(F_{i}\right)$, where $\operatorname{SEP}\left(F_{i}\right)$ is the set of solutions to the equilibrium problems, that is,

$$
F_{i}\left(x^{*}, y\right) \geq 0, \quad \forall y \in C .
$$

(C) 2012 Onjai-uea et al; licensee Springer. This is an Open Access article distributed under the terms of the Creative Commons Attribution License (http://creativecommons.org/licenses/by/2.0), which permits unrestricted use, distribution, and reproduction in any medium, provided the original work is properly cited. 
If $N=1$, then the problem (1.2) is reduced to the equilibrium problems.

If $N=1$ and $F\left(x^{*}, y\right)=\left\langle B x^{*}, y-x^{*}\right\rangle$, then the problem (1.2) is reduced to the variational inequality problems of finding $x^{*} \in C$ such that

$$
\left\langle B x^{*}, y-x^{*}\right\rangle \geq 0, \quad \forall y \in C .
$$

The set of solutions of (1.4) is denoted by $V I(C, B)$.

Many problems in applied sciences, such as monotone inclusion problems, saddle point problems, optimization problems, variational inequality problems, Nash equilibrium problems, and equilibrium problems as special cases. Some methods have been proposed to solve $V I(C, B), E P(F)$, and $S E P\left(F_{i}\right)$; see, for example [1-22] and references therein. The above formulations (1.2) extends this formulism to such problems, covering in particular various forms of feasibility problems [23,24].

Let $P_{C}$ be the metric projection of $H$ onto the closed convex subset $C$. Let $S: C \rightarrow C$ be a nonexpansive mapping, that is, $\|S x-S y\| \leq\|x-y\|$ for all $x, y \in C$. The set of fixed points of $S$ is denoted by $F(S)=\{x \in C: S x=x\}$. If $C \subset H$ is nonempty, bounded, closed and convex and $S$ is a nonexpansive mapping of $C$ into itself, then $F(S)$ is nonempty; see, for example, [25,26]. A mapping $f: C \rightarrow C$ is a contraction on $C$ if there exists a constant $\eta \in(0,1)$ such that $\|f(x)-f(y)\| \leq \eta\|x-y\|$ for all $x, y \in C$.

Definition 1.1. Let $B: C \rightarrow H$ be nonlinear mappings. Then $B$ is called

(1) monotone if $\langle B x-B y, x-y\rangle \geq 0, \forall x, y \in C$,

(2) $\xi$-inverse-strongly monotone if there exists a constant $\xi>0$ such that

$$
\langle B x-B y, x-y\rangle \geq \xi\|B x-B y\|^{2}, \quad \forall x, y \in C .
$$

(3) A set-valued mapping $Q: H \rightarrow 2^{H}$ is called monotone if for all $x, y \in H, f \in Q x$ and $g \in Q y$ imply $\langle x-y, f-g\rangle \geq 0$. A monotone mapping $Q: H \rightarrow 2^{H}$ is called maximal monotone, if it is monotone and if for any $(x, f) \in H \times H$

$$
\langle x-y, f-g\rangle \geq 0, \quad \forall(y, g) \in \operatorname{Graph}(Q)
$$

(the graph of mapping $Q$ ) implies that $f \in Q x$.

A typical problem is to minimize a quadratic function over the set of fixed points of a nonexpansive mapping defined on a real Hilbert space $H$ :

$$
\min _{x \in F}\left[\frac{1}{2}\langle A x, x\rangle-\langle x, b\rangle\right],
$$

where $F$ is the fixed point set of a nonexpansive mapping $S$ defined on $H$ and $b$ is a given point in $H$.

A linear bounded operator $A$ is strong positive if there exists a constant $\bar{\gamma}>0$ with the property

$$
\langle A x, x\rangle \geq \bar{\gamma}\|x\|^{2}, \quad \forall x \in H .
$$

Marino and $\mathrm{Xu}$ [27] introduced a new iterative scheme by the viscosity approximation method:

$$
x_{n+1}=\varepsilon_{n} \gamma f\left(x_{n}\right)+\left(1-\varepsilon_{n} A\right) S x_{n} .
$$


They proved that the sequences $\left\{x_{n}\right\}$ generated by (1.5) converges strongly to the unique solution of the variational inequality:

$$
\langle\gamma f z-A z, x-z\rangle \leq 0, \quad \forall x \in F(S),
$$

which is the optimality condition for the minimization problem:

$$
\min _{x \in F(S)}\left[\frac{1}{2}\langle A x, x\rangle-h(x)\right],
$$

where $h$ is a potential function for $\gamma f$.

In order to find a common element of the set of fixed points of a nonexpansive mapping and the set of solutions of variational inequalities for a $\xi$-inverse-strongly monotone mapping, Takahashi and Toyoda [28] introduced the following iterative scheme:

$$
\left\{\begin{array}{l}
x_{0} \in C \text { chosen arbitrary, } \\
x_{n+1}=\gamma_{n} x_{n}+\left(1-\gamma_{n}\right) S P_{C}\left(x_{n}-\alpha_{n} B x_{n}\right), \quad \forall n \geq 0,
\end{array}\right.
$$

where $B$ is a $\xi$-inverse-strongly monotone mapping, $\left\{\gamma_{n}\right\}$ is a sequence in $(0,1)$, and $\left\{\alpha_{n}\right\}$ is a sequence in $(0,2 \xi)$. They showed that if $F(S) \cap V I(C, B)$ is nonempty, then the sequence $\left\{x_{n}\right\}$ generated by (1.7) converges weakly to some $z \in F(S) \cap V I(C, B)$.

In order to find a common element of $F(S) \cap V I(C, B)$, let $S: H \rightarrow H$ be a nonexpansive mapping, Yamada [29] introduced the following iterative scheme called the hybrid steepest descent method:

$$
x_{n+1}=S x_{n}-\alpha_{n} \mu B S x_{n}, \quad \forall n \geq 1,
$$

where $x_{1}=x \in H,\left\{\alpha_{n}\right\} \subset(0,1)$, let $B: H \rightarrow H$ be a strongly monotone and Lipschitz continuous mapping and $\mu$ is a positive real number. He proved that the sequence $\left\{x_{n}\right\}$ generated by (1.8) converges strongly to the unique solution of the $F(S) \cap V I(C, B)$.

Let $C$ be a nonempty closed convex subset of $H$. Given $r>0$ the operators $J_{r}^{F}: H \rightarrow C$ defined by

$$
J_{r}^{F}(x)=\left\{z \in C: F(z, y)+\frac{1}{r}\langle y-z, z-x\rangle \geq 0, \quad \forall y \in C\right\},
$$

is called the resolvent of $F$ (see [3]). It is shown in [3] that under suitable hypotheses on $F$ (to be stated precisely in Section 2), $J_{r}^{F}: H \rightarrow C$ is single-valued and firmly nonexpansive and satisfied

$$
F\left(J_{r}^{F}\right)=E P(F), \quad \forall r>0 .
$$

Using the result, in 2009, Colao et al. [10] introduced and considered an implicit iterative scheme for finding a common element of the set of solutions of the system equilibrium problems and the set of common fixed points of an infinite family of nonexpansive mappings on $C$. Starting with an arbitrary initial $x_{0} \in C$ and defining a sequence $\left\{z_{n}\right\}$ recursively by

$$
x_{n}=\varepsilon_{n} \gamma f\left(x_{n}\right)+\left(1-\varepsilon_{n} A\right) W_{n} J_{r_{M, n}}^{F_{M}} J_{r_{M-1, n}}^{F_{M-1}} J_{r_{M-2, n}}^{F_{M-2}} \cdots J_{r_{2, n}}^{F_{2}} J_{r_{1, n}}^{F_{1}} x_{n},
$$

where $\left\{\epsilon_{n}\right\}$ be a sequences in $(0,1)$. It is proved [10] that under certain appropriate conditions imposed on $\left\{\epsilon_{n}\right\}$ and $\left\{r_{n}\right\}$, the sequence $\left\{x_{n}\right\}$ generated by (1.9) converges 
strongly to $z \in \cap_{n=1}^{\infty} F\left(T_{n}\right) \cap\left(\cap_{k=1}^{M} S E P\left(F_{k}\right)\right)$, where $z$ is the unique solution of the variational inequality and which is the optimality condition for the minimization problem.

In 2010, Colao and Marino [30] introduced the following explicit viscosity scheme with respect to $W$-mappings for an infinite family of nonexpansive mappings

$$
x_{n+1}=\varepsilon_{n} \gamma f\left(x_{n}\right)+\beta_{n} x_{n}+\left(\left(1-\beta_{n}\right) I-\varepsilon_{n} A\right) W_{n} J_{r_{n}}^{F} x_{n} .
$$

They prove that sequence $\left\{x_{n}\right\}$ and $\left\{J_{r_{n}}^{F}\right\}$ converge strongly to $z \in \cap_{n=1}^{\infty} F\left(T_{n}\right) \cap E P(F)$, where $z$ is an equilibrium point for $F$ and is the unique solution of the variational inequality:

$$
\langle\gamma f z-A z, x-z\rangle \leq 0, \quad \forall x \in \cap_{n=1}^{\infty} F\left(T_{n}\right) \cap E P(F)
$$

or, equivalently, the unique solution of the minimization problem

$$
\min _{x \in \cap_{n=1}^{\infty} F\left(T_{n}\right) \cap E P(F)}\left[\frac{1}{2}\langle A x, x\rangle-h(x)\right],
$$

where $h$ is a potential function for $\gamma f$. Recently, Chantarangsi et al. [11] introduced some iterative processes based on the viscosity hybrid steepest descent method for finding a common solutions of a generalized mixed equilibrium problem, the set of fixed points of a nonexpansive mapping and the set of solutions of variational inequality problem in a real Hilbert space.

In this article, motivated by above results, we introduce an iterative scheme for finding a common element of the set of solutions of system of equilibrium problems, the set of fixed points of an infinite family of nonexpansive mapping, and the set of solutions of variational inequality problems for inverse strongly monotone mapping in a real Hilbert space by using a new hybrid steepest descent methods. The results shown in this article improve and extend the recent ones announced by many others.

\section{Preliminaries}

Let $H$ be a real Hilbert space, when $\left\{x_{n}\right\}$ is a sequence in $H$, we denote strong convergence of $\left\{x_{n}\right\}$ to $x \in H$ by $x_{n} \rightarrow x$ and weak convergence by $x_{n} \rightarrow x$. Let $C$ be nonempty closed convex subset of $H$. The nearest point projection $P_{C}: H \rightarrow C$ defined from $H$ onto $C$ is the function which assigns to each $x \in H$ its nearest point denoted by $P_{C} x$ in $C$. Thus, $P_{C} x$ is the unique point in $C$ such that $\left\|x-P_{C} x\right\| \leq\|x-y\|, \forall y \in C$. It easy to see that $P_{C}$ is nonexpansive and

$$
x^{*} \in \operatorname{VI}(C, B) \Leftrightarrow x^{*}=P_{C}\left(x^{*}-\lambda B x^{*}\right), \quad \lambda>0 .
$$

Lemma 2.1. [26] Let $H$ be a Hilbert space, let $C$ be a nonempty closed convex subset of $H$. Let $\xi>0$ and let $A: C \rightarrow H$ be $\xi$-inverse strongly monotone. If $0<\mathrm{\varrho} \leq 2 \xi$, then $I$ $\mathrm{Q} B$ is a nonexpansive mapping of $C$ into $H$.

Lemma 2.2. [26] Let $H$ be a real Hilbert spaces, there hold the following identities:

(i) for each $x \in H$ and $x^{*} \in C, x^{*}=P_{C} x \Leftrightarrow\left\langle x-x^{*}, y-x^{*}\right\rangle \leq 0$ for all $y \in C$;

(ii) $P_{C}: H \rightarrow C$ is nonexpansive, that is, $\left\|P_{C} x-P_{C} y\right\| \leq\|x-y\|$ for all $x, y \in H$;

(iii) $P_{C}$ is firmly nonexpansive, that is, $\left\|P_{C} x-P_{C} y\right\|^{2} \leq\left\langle P_{C} x-P_{C} y, x-y\right\rangle$ for all $x, y$

$\in H$; 
(iv) $\|t x+(1-t) y\|^{2}=t|| x\left\|^{2}+(1-t)\right\| y\left\|^{2}-t(1-t)\right\| x-y \|^{2}, \forall t \in[0,1]$, for all $x, y$

$\in H$;

(v) $\|x+y\|^{2} \leq\|x\|^{2}+2\langle y, x+y\rangle$.

Lemma 2.3. [31]Each Hilbert space H satisfies Opial's condition, that is, for any sequence $\left\{x_{n}\right\} \subset H$ with $x_{n} \rightarrow x$, the inequality

$$
\liminf _{n \rightarrow \infty}\left\|x_{n}-x\right\|<\liminf _{n \rightarrow \infty}\left\|x_{n}-y\right\|,
$$

hold for each $y \in H$ with $y \neq x$.

Lemma 2.4. [27] Let $C$ be a nonempty closed convex subset of $H$ and let $f$ be a contraction of $H$ into itself with $\eta \in(0,1)$, and $A$ be a strongly positive linear bounded operator on $H$ with coefficient $\bar{\gamma}>0$. Then, for $0<\gamma<\frac{\bar{\gamma}}{\eta}$,

$$
\left\langle x-\gamma_{,}(A-\gamma f) x-(A-\gamma f) \gamma\right\rangle \geq(\bar{\gamma}-\eta \gamma)\|x-\gamma\|^{2}, \quad \forall x, y \in H .
$$

That is, $A-\gamma f$ is a strongly monotone with coefficient $\bar{\gamma}-\gamma \eta$.

Lemma 2.5. [27]Assume $A$ be a strongly positive linear bounded operator on $H$ with coefficient $\bar{\gamma}>0$ and $0<\rho \leq\|A\|^{-1}$. Then $\|I-\rho A\| \leq 1-\rho \bar{\gamma}$.

Throughout this article, we assume that a bifunction $F: C \times C \rightarrow \mathbb{R}$ satisfies the following conditions:

(A1) $F(x, x)=0$ for all $x \in C$;

(A2) $F$ is monotone, i.e., $F(x, y)+F(y, x) \leq 0$ for all $x, y \in C$;

(A3) for each $x, y, z \in C, \lim _{t \downarrow 0} F(t z+(1-t) x, y) \leq F(x, y)$;

(A4) for each $x \in C, y \mapsto F(x, y)$ is convex and lower semicontinuous.

Then, we have the following lemmas.

Lemma 2.6. [1] Let $C$ be a nonempty closed convex subset of $H$ and let $F$ be a bifunction of $C \times C$ into $\mathbb{R}$ satisfying (A1)-(A4). Let $r>0$ and $x \in H$. Then, there exists $z \in C$ such that

$$
F(z, y)+\frac{1}{r}\langle y-z, z-x\rangle \geq 0, \quad \forall y \in C .
$$

Lemma 2.7. [3] Assume that $F: C \times C \rightarrow \mathbb{R}$ satisfies (A1)-(A4). For $r>0$ and $x \in H$, define a mapping $J_{r}^{H}: H \rightarrow$ Cas follows:

$$
J_{r}^{F}(x)=\left\{z \in C: F(z, y)+\frac{1}{r}\langle y-z, z-x\rangle \geq 0, \quad \forall y \in C\right\}
$$

for all $z \in H$. Then, the following hold:

(1) $J_{r}^{F}$ is single-valued;

(2) $J_{r}^{F}$ is firmly nonexpansive, that is, for any $x, y \in H$,

$$
\left\|J_{r}^{F} x-J_{r}^{F} y\right\|^{2} \leq\left\langle J_{r}^{F} x-J_{r}^{F} y, x-\gamma\right\rangle ;
$$


(3) $F\left(J_{r}^{F}\right)=E P(F)$;

(4) $E P(F)$ is closed and convex.

Lemma 2.8. [32] Let $\left\{x_{n}\right\}$ and $\left\{l_{n}\right\}$ be bounded sequences in a Banach space $X$ and let $\left\{\beta_{n}\right\}$ be a sequence in $[0,1]$ with $0<\lim _{\inf _{n \rightarrow \infty}} \beta_{n} \leq \lim \sup _{n \rightarrow \infty} \beta_{n}<1$. Suppose $x_{n+1}=$ $\left(1-\beta_{n}\right) l_{n}+\beta_{n} x_{n}$ for all integers $n \geq 0$ and $\lim \sup _{n \rightarrow \infty}\left(|| l_{n+1}-l_{n} \|-|| x_{n+1}-x_{n}||\right) \leq 0$. Then, $\lim _{n \rightarrow \infty}\left\|l_{n}-x_{n}\right\|=0$.

Lemma 2.9. [33]Assume $\left\{a_{n}\right\}$ is a sequence of nonnegative real numbers such that

$$
a_{n+1} \leq\left(1-b_{n}\right) a_{n}+c_{n}, n \geq 0,
$$

where $\left\{b_{n}\right\}$ is a sequence in $(0,1)$ and $\left\{c_{n}\right\}$ is a sequence in $\mathbb{R}$ such that

(1) $\sum_{n=1}^{\infty} b_{n}=\infty$,

(2) $\lim \sup _{n \rightarrow \infty} \frac{c_{n}}{b_{n}} \leq$ or $\sum_{n=1}^{\infty}\left|c_{n}\right|<\infty$,

Then, $\lim _{n \rightarrow \infty} a_{n}=0$.

\section{Main results}

Let $C$ be a nonempty closed convex subset of a real Hilbert space $H$. Let $\left\{T_{n}\right\}_{n=1}^{\infty}$ be a family of infinitely of nonexpansive mappings of $C$ into itself and let $\left\{\mu_{n}\right\}_{n=1}^{\infty}$ be a sequence of nonnegative numbers in [0,1]. For any $n \geq 1$, define a mapping $W_{n}: C \rightarrow C$ as follows:

$$
\begin{aligned}
U_{n, n+1} & =I, \\
U_{n, n} & =\mu_{n} T_{n} U_{n, n+1}+\left(1-\mu_{n}\right) I, \\
U_{n, n-1} & =\mu_{n-1} T_{n-1} U_{n, n}+\left(1-\mu_{n-1}\right) I, \\
& \vdots \\
U_{n, k} & =\mu_{k} T_{k} U_{n, k+1}+\left(1-\mu_{k}\right) I, \\
U_{n, k-1} & =\mu_{k-1} T_{k-1} U_{n, k}+\left(1-\mu_{k-1}\right) I, \\
& \vdots \\
U_{n, 2} & =\mu_{2} T_{2} U_{n, 3}+\left(1-\mu_{2}\right) I, \\
W_{n} & =U_{n, 1}=\mu_{1} T_{1} U_{n, 2}+\left(1-\mu_{1}\right) I,
\end{aligned}
$$

such a mappings $W_{n}$ is nonexpansive from $C$ to $C$ and it is called the $W$-mapping generated by $T_{1}, T_{2}, \ldots, T_{n}$ and $\mu_{1}, \mu_{2}, \ldots, \mu_{n}$ (see [34]).

Lemma 3.1. $[34,35]$ Let $C$ be a nonempty closed convex subset of a real Hilbert space H. Let $T_{1}, T_{2}, \ldots$, be an infinite family of nonexpansive mappings of $C$ into itself such that $\cap_{n=1}^{\infty} F\left(T_{n}\right) \neq \emptyset$, let $\mu_{1}, \mu_{2}, \ldots$ be real numbers such that $0 \leq \mu_{n} \leq b<1$ for every $n \geq 1$. Then,

(1) for every $x \in C$ and $k \in \mathbb{N}$, the limit $\lim _{n \rightarrow \infty} U_{n, k} x$ exists;

(2) the mapping $W$ of $C$ into itself as follows:

$$
W x=\lim _{n \rightarrow \infty} W_{n} x=\lim _{n \rightarrow \infty} U_{n, 1} x, \quad x \in C
$$


is a nonexpansive mapping satisfying $F(W)=\cap_{n=1}^{\infty} F\left(T_{n}\right)$, which it is called the $W$ mapping generated by $T_{1}, T_{2}, \ldots$ and $\mu_{1}, \mu_{2}, \ldots$;

(3) $F\left(W_{n}\right)=\cap_{n=1}^{\infty} F\left(T_{n}\right)$, for each $n \geq 1$;

(4) If $E$ is any bounded subset of $C$, then $\lim _{n \rightarrow \infty} \sup _{x \in E}\left\|W x-W_{n} x\right\|=0$.

Theorem 3.2. Let $C$ be a nonempty closed convex subset of a real Hilbert space $H$, let $F_{k}, k \in\{1,2,3, \ldots, M\}$ be a bifunction from $C \times C$ to $\mathbb{R}$ satisfying (A1)-(A4), let $\left\{T_{n}\right\}$ be an infinite family of nonexpansive mappings of $C$ into itself and let $B$ be $\xi$-inverse strongly monotone such that

$$
\Theta:=\cap_{n=1}^{\infty} F\left(T_{n}\right) \cap\left(\cap_{k=1}^{M} \operatorname{SEP}\left(F_{k}\right)\right) \cap \operatorname{VI}(C, B) \neq \emptyset .
$$

Let $f$ be a contraction of $H$ into itself with $\eta \in(0,1)$ and let $A$ be a strongly positive linear bounded operator on $H$ with coefficient $\bar{\gamma}>0$ and $0<\gamma<\frac{\bar{\gamma}}{\eta}$. Let $\left\{x_{n}\right\},\left\{y_{n}\right\}$ and $\left\{u_{n}\right\}$ be sequences generated by

$$
\left\{\begin{array}{l}
x_{1}=x \in C \text { chosen arbitrary, } \\
y_{n}=\left(1-\delta_{n}\right) x_{n}+\delta_{n} P_{C}\left(x_{n}-\alpha_{n} B x_{n}\right), \\
u_{n}=J_{r_{M, n}}^{F_{M}} J_{r_{M-1, n}}^{F_{M-2}} J_{r_{M-2, n}}^{F_{M}} \ldots J_{r_{2, n}}^{F_{2}} F_{r_{1, n}}^{F_{1}} y_{n} \\
x_{n+1}=\varepsilon_{n} \gamma f\left(u_{n}\right)+\beta_{n} x_{n}+\left(\left(1-\beta_{n}\right) I-\varepsilon_{n} A\right) P_{C}\left(W_{n} u_{n}-\lambda_{n} B W_{n} u_{n}\right), \quad \forall n \geq 1,
\end{array}\right.
$$

where $\left\{W_{n}\right\}$ is the sequence generated by (3.1) and $\left\{\epsilon_{n}\right\},\left\{\beta_{n}\right\}$ are two sequences in $(0,1)$ and $\left\{r_{k, n}\right\}, k \in\{1,2,3, \ldots, M\}$ are a real sequence in $(0, \infty)$ satisfy the following conditions:

(C1) $\lim _{n \rightarrow \infty} \epsilon_{n}=0$ and $\sum_{n=1}^{\infty} \varepsilon_{n}=\infty$,

(C2) $0<\lim _{\inf _{n \rightarrow \infty}} \beta_{n} \leq \lim \sup _{n \rightarrow \infty} \beta_{n}<1$,

(C3) $\left\{\alpha_{n}\right\},\left\{\lambda_{n}\right\} \subset[e, g] \subset(0,2 \xi), \lim _{n \rightarrow \infty} \alpha_{n}=0$ and $\lim _{n \rightarrow \infty} \lambda_{n}=0$,

(C4) $\left\{\delta_{n}\right\} \subset[0, b]$, for some $b \in(0,1)$ and $\lim _{n \rightarrow \infty}\left|\delta_{n+1}-\delta_{n}\right|=0$,

(C5) $\lim \inf _{n \rightarrow \infty} r_{k, n}>0$ and $\lim _{n \rightarrow \infty}\left|r_{k, n+1}-r_{k, n}\right|=0$ for each $k \in\{1,2,3, \ldots, M\}$.

Then, $\left\{x_{n}\right\}$ and $\left\{u_{n}\right\}$ converge strongly to a point $z \in \Theta$, which is the unique solution of the variational inequality

$$
\langle(A-\gamma f) z, x-z\rangle \geq 0, \quad \forall x \in \Theta .
$$

Equivalently, we have $z=P_{\Theta}(I-A+\gamma f)(z)$.

Proof. From the restrictions on control sequence, without loss of generality, that $\epsilon_{n} \leq$ $\left(1-\beta_{n}\right)\|A\|^{-1}$ for all $n \geq 1$. From Lemma 2.5, we know that if $0 \leq \rho \leq\|A\|^{-1}$, then $\|I-\rho A\| \leq 1-\rho \bar{\gamma}$. We will assume that $\|I-A\| \leq 1-\bar{\gamma}$. Since $A$ is a strongly positive bounded linear operator on $H$, we have

$$
\|A\|=\sup \{|\langle A x, x\rangle|: x \in H,\|x\|=1\} .
$$

Observe that

$$
\left\langle\left(\left(1-\beta_{n}\right) I-\varepsilon_{n} A\right) x, x\right\rangle=1-\beta_{n}-\varepsilon_{n}\langle A x, x\rangle \geq 1-\beta_{n}-\varepsilon_{n}\|A\| \geq 0,
$$


this show that $\left(1-\beta_{n}\right) I-\epsilon_{n} A$ is positive. It follows that

$$
\begin{aligned}
\left\|\left(1-\beta_{n}\right) I-\varepsilon_{n} A\right\| & =\sup \left\{\left|\left\langle\left(\left(1-\beta_{n}\right) I-\varepsilon_{n} A\right) x, x\right\rangle\right|: x \in H,\|x\|=1\right\} \\
& =\sup \left\{1-\beta_{n}-\varepsilon_{n}\langle A x, x\rangle: x \in H,\|x\|=1\right\} \\
& \leq 1-\beta_{n}-\varepsilon_{n} \bar{\gamma} .
\end{aligned}
$$

We divide the proof of Theorem 3.2 into seven steps.

Step 1. We show that the mapping $P_{\Theta}(\gamma f+(I-A))$ has a unique fixed point.

Since $f$ be a contraction of $C$ into itself with coefficient $\eta \in(0,1)$. Then, we have

$$
\begin{aligned}
\left\|P_{\Theta}(\gamma f+(I-A))(x)-P_{\Theta}(\gamma f+(I-A))(\gamma)\right\| & \leq\|(\gamma f+(I-A))(x)-(\gamma f+(I-A))(\gamma)\| \\
& \leq \gamma\|f(x)-f(\gamma)\|+\|I-A\|\|x-\gamma\| \\
& \leq \gamma \eta\|x-\gamma\|+(1-\bar{\gamma})\|x-\gamma\| \\
& =(1-(\bar{\gamma}-\eta \gamma))\|x-\gamma\|, \quad \forall x, y \in C .
\end{aligned}
$$

Since $0<1-(\bar{\gamma}-\eta \gamma)<1$, it follows that $P_{\Theta}(\gamma f+(I-A))$ is a contraction of $C$ into itself. Therefore, by the Banach Contraction Mapping Principle, has a unique fixed point, say $z \in C$, that is,

$$
z=P_{\Theta}(\gamma f+(I-A))(z) .
$$

Step 2. We show that $W_{n}-\lambda_{n} B W_{n}$ is nonexpansive.

For all $x, y \in C$, let $W_{n}$ is the sequence defined by (3.1) and $\lambda_{n} \in(0,2 \xi)$, we obtain $W_{n}-\lambda_{n} B W_{n}$ is a nonexpansive. Indeed,

$$
\begin{aligned}
& \left\|\left(W_{n}-\lambda_{n} B W_{n}\right) x-\left(W_{n}-\lambda_{n} B W_{n}\right) y\right\|^{2} \\
= & \left\|\left(W_{n} x-W_{n} y\right)-\lambda_{n}\left(B W_{n} x-B W_{n} y\right)\right\|^{2} \\
= & \left\|W_{n} x-W_{n} y\right\|^{2}-2 \lambda_{n}\left\langle W_{n} x-W_{n} y, B W_{n} x-B W_{n} y\right\rangle+\lambda_{n}^{2}\left\|B W_{n} x-B W_{n} y\right\|^{2} \\
\leq & \|x-y\|^{2}-2 \lambda_{n} \xi\left\|B W_{n} x-B W_{n} y\right\|+\lambda_{n}^{2}\left\|B W_{n} x-B W_{n} y\right\|^{2} \\
= & \|x-y\|^{2}-\lambda_{n}\left(\lambda_{n}-2 \xi\right)\left\|B W_{n} x-B W_{n} \gamma\right\|^{2} \\
\leq & \|x-y\|^{2}
\end{aligned}
$$

which implies that $W_{n}-\lambda_{n} B W_{n}$ is a nonexpansive.

Step 3. We show that the sequence $\left\{x_{n}\right\}$ is bounded.

In fact, let $\tilde{x} \in \Theta$, then

$$
\tilde{x}=P_{C}\left(\tilde{x}-\alpha_{n} B \tilde{x}\right) .
$$

Setting $v_{n}=P_{C}\left(x_{n}-\alpha_{n} B x_{n}\right)$ and $I-\alpha_{n} B$ is a nonexpansive mapping (Lemma 2.1), we obtain

$$
\begin{aligned}
\left\|v_{n}-\tilde{x}\right\| & =\left\|P_{C}\left(x_{n}-\alpha_{n} B x_{n}\right)-P_{C}\left(\tilde{x}-\alpha_{n} B \tilde{x}\right)\right\| \\
& \leq\left\|\left(x_{n}-\alpha_{n} B x_{n}\right)-\left(\tilde{x}-\alpha_{n} B \tilde{x}\right)\right\| \\
& =\left\|\left(I-\alpha_{n} B\right) x_{n}-\left(I-\alpha_{n} B\right) \tilde{x}\right\| \\
& \leq\left\|x_{n}-\tilde{x}\right\|
\end{aligned}
$$


and

$$
\begin{aligned}
\left\|y_{n}-\tilde{x}\right\| & \leq\left(1-\delta_{n}\right)\left\|x_{n}-\tilde{x}\right\|+\delta_{n}\left\|v_{n}-\tilde{x}\right\| \\
& \leq\left(1-\delta_{n}\right)\left\|x_{n}-\tilde{x}\right\|+\delta_{n}\left\|x_{n}-\tilde{x}\right\| \\
& =\left\|x_{n}-\tilde{x}\right\| .
\end{aligned}
$$

Let $\Im_{n}^{k}=J_{r_{k, n}}^{F_{k}} J_{r_{k-1, n}}^{F_{k-1}} J_{r_{k-2, n}}^{F_{k-2}} \ldots J_{r_{2, n}}^{F_{2}} J_{r_{1, n}}^{F_{1}}$ for $k \in\{1,2,3, \ldots, M\}$ and $\Im_{n}^{0}=I$ for all $n$. Because $J_{r_{k, n}}^{F_{k}}$ is nonexpansive for each $k=1,2,3, \ldots, M, \tilde{x}=\Im_{n}^{k} \tilde{x}$ and (3.7), we note that $u_{n}=\mathfrak{\Im}_{n}^{M} y_{n}$. It follows that

$$
\left\|u_{n}-\tilde{x}\right\|=\left\|\Im_{n}^{M} y_{n}-\Im_{n}^{M} \tilde{x}\right\| \leq\left\|y_{n}-\tilde{x}\right\| \leq\left\|x_{n}-\tilde{x}\right\| .
$$

Let $e_{n}=P_{C}\left(W_{n} u_{n}-\lambda_{n} B W_{n} u_{n}\right)$, we can prove that

$$
\begin{aligned}
\left\|e_{n}-\tilde{x}\right\| & =\left\|P_{C}\left(W_{n} u_{n}-\lambda_{n} B W_{n} u_{n}\right)-P_{C}\left(W_{n} \tilde{x}-\lambda_{n} B W_{n} \tilde{x}\right)\right\| \\
& \leq\left\|\left(W_{n} u_{n}-\lambda_{n} B W_{n} u_{n}\right)-\left(W_{n} \tilde{x}-\lambda_{n} B W_{n} \tilde{x}\right)\right\| \\
& =\left\|\left(W_{n}-\lambda_{n} B W_{n}\right) u_{n}-\left(W_{n}-\lambda_{n} B W_{n}\right) \tilde{x}\right\| \\
& \leq\left\|u_{n}-\tilde{x}\right\| \leq\left\|x_{n}-\tilde{x}\right\|,
\end{aligned}
$$

which yields that

$$
\begin{aligned}
\left\|x_{n+1}-\tilde{x}\right\| & =\left\|\varepsilon_{n}\left(\gamma f\left(u_{n}\right)-A \tilde{x}\right)+\beta_{n}\left(x_{n}-\tilde{x}\right)+\left(\left(1-\beta_{n}\right) I-\varepsilon_{n} A\right)\left(e_{n}-\tilde{x}\right)\right\| \\
& \leq \varepsilon_{n}\left\|\gamma f\left(u_{n}\right)-A \tilde{x}\right\|+\beta_{n}\left\|x_{n}-\tilde{x}\right\|+\left\|\left(1-\beta_{n}\right) I-\varepsilon_{n} A\right\|\left\|e_{n}-\tilde{x}\right\| \\
& \leq \varepsilon_{n} \gamma\left\|f\left(u_{n}\right)-f(\tilde{x})\right\|+\varepsilon_{n}\|\gamma f(\tilde{x})-A \tilde{x}\|+\beta_{n}\left\|x_{n}-\tilde{x}\right\|+\left(1-\beta_{n}-\varepsilon_{n} \bar{\gamma}\right)\left\|e_{n}-\tilde{x}\right\| \\
& \leq \varepsilon_{n} \gamma \eta\left\|u_{n}-\tilde{x}\right\|+\varepsilon_{n}\|\gamma f(\tilde{x})-A \tilde{x}\|+\beta_{n}\left\|x_{n}-\tilde{x}\right\|+\left(1-\beta_{n}-\varepsilon_{n} \bar{\gamma}\right)\left\|x_{n}-\tilde{x}\right\| \\
& \leq \varepsilon_{n} \gamma \eta\left\|x_{n}-\tilde{x}\right\|+\varepsilon_{n}\|\gamma f(\tilde{x})-A \tilde{x}\|+\beta_{n}\left\|x_{n}-\tilde{x}\right\|+\left(1-\beta_{n}-\varepsilon_{n} \bar{\gamma}\right)\left\|x_{n}-\tilde{x}\right\| \\
& =\left(1-(\bar{\gamma}-\gamma \eta) \varepsilon_{n}\right)\left\|x_{n}-\tilde{x}\right\|+\frac{(\bar{\gamma}-\gamma \eta) \varepsilon_{n}}{(\bar{\gamma}-\gamma \eta)}\|\gamma f(\tilde{x})-A \tilde{x}\| .
\end{aligned}
$$

By induction, we have

$$
\left\|x_{n}-\tilde{x}\right\| \leq \max \left\{\left\|x_{1}-\tilde{x}\right\|, \frac{\|\gamma f(\tilde{x})-A \tilde{x}\|}{\bar{\gamma}-\gamma \eta}\right\}, \quad \forall n \in \mathbb{N} .
$$

This implies that $\left\{x_{n}\right\}$ is bounded, and hence so are $\left\{u_{n}\right\},\left\{e_{n}\right\},\left\{y_{n}\right\},\left\{B W_{n} u_{n}\right\},\left\{B x_{n}\right\}$, $\left\{A e_{n}\right\},\left\{v_{n}-x_{n}\right\}$, and $\left\{f\left(u_{n}\right)\right\}$.

Step 4. We show that $\lim _{n \rightarrow \infty}\left\|x_{n+1}-x_{n}\right\|=0$.

We claim that if $\omega_{n}$ be a bounded sequence in $C$, then

$$
\lim _{n \rightarrow \infty}\left\|\Im_{n}^{k} \omega_{n}-\Im_{n+1}^{k} \omega_{n}\right\|=0
$$

for every $k \in\{1,2,3, \ldots, M\}$. From Step 2 of the proof of Theorem 3.1 in [10], we have that for $k \in\{1,2,3, \ldots, M\}$,

$$
\lim _{n \rightarrow \infty}\left\|J_{r_{k, n+1}}^{F_{k}} \omega_{n}-J_{r_{k, n}}^{F_{k}} \omega_{n}\right\|=0
$$


Note that for every $k \in\{1,2,3, \ldots, M\}$, we obtain

$$
\Im_{n}^{k}=J_{r_{k, n}}^{F_{k}} J_{r_{k-1, n}}^{F_{k-1}} J_{r_{k-2, n}}^{F_{k-2}} \ldots J_{r_{2, n}}^{F_{2}} J_{r_{1, n}}^{F_{1}}=J_{r_{k, n}}^{F_{k}} \Im_{n}^{k-1} .
$$

Thus,

$$
\begin{aligned}
& \left\|\Im_{n}^{k} \omega_{n}-\Im_{n+1}^{k} \omega_{n}\right\| \\
= & \left\|J_{r_{k, n}}^{F_{k}} \Im_{n}^{k-1} \omega_{n}-J_{r_{k, n+1}}^{F_{k}} \Im_{n+1}^{k-1} \omega_{n}\right\| \\
\leq & \left\|J_{r_{k, n}}^{F_{k}} \Im_{n}^{k-1} \omega_{n}-J_{r_{k, n+1}}^{F_{k}} \Im_{n}^{k-1} \omega_{n}\right\|+\left\|J_{r_{k, n+1}}^{F_{k}} \Im_{n}^{k-1} \omega_{n}-J_{r_{k, n+1}}^{F_{k}} \Im_{n+1}^{k-1} \omega_{n}\right\| \\
\leq & \left\|J_{r_{k, n}}^{F_{k}} \Im_{n}^{k-1} \omega_{n}-J_{r_{k, n+1}}^{F_{k}} \Im_{n}^{k-1} \omega_{n}\right\|+\left\|\Im_{n}^{k-1} \omega_{n}-\Im_{n+1}^{k-1} \omega_{n}\right\| \\
\leq & \left\|J_{r_{k, n}}^{F_{k}} \Im_{n}^{k-1} \omega_{n}-J_{r_{k, n+1}}^{F_{k}} \Im_{n}^{k-1} \omega_{n}\right\|+\left\|J_{r_{k-1, n}}^{F_{k-1}} \Im_{n}^{k-2} \omega_{n}-J_{r_{k-1, n+1}}^{F_{k-1}} \Im_{n}^{k-2} \omega_{n}\right\| \\
& +\left\|\Im_{n}^{k-2} \omega_{n}-\Im_{n+1}^{k-2} \omega_{n}\right\| \\
\leq & \left\|J_{r_{k, n}}^{F_{k}} \Im_{n}^{k-1} \omega_{n}-J_{r_{k, n+1}}^{F_{k}} \Im_{n}^{k-1} \omega_{n}\right\|+\left\|J_{r_{k-1, n}}^{F_{k-1}} \Im_{n}^{k-2} \omega_{n}-J_{r_{k-1, n+1}}^{F_{k-1}} \Im_{n}^{k-2} \omega_{n}\right\| \\
& +\ldots+\left\|J_{r_{2, n}}^{F_{2}} \Im_{n}^{1} \omega_{n}-J_{r_{2, n+1}}^{F_{2}} \Im_{n}^{1} \omega_{n}\right\|+\left\|J_{r_{1, n}}^{F_{1}} \omega_{n}-J_{r_{1, n+1}}^{F_{1}} \omega_{n}\right\| .
\end{aligned}
$$

Now, apply (3.12) to conclude (3.11).

Since $T_{n}$ and $U_{n, n}$ are nonexpansive, we have

$$
\begin{aligned}
\left\|W_{n+1} x_{n}-W_{n} x_{n}\right\| & =\left\|\mu_{1} T_{1} U_{n+1,2} x_{n}-\mu_{1} T_{1} U_{n, 2} x_{n}\right\| \\
& \leq \mu_{1}\left\|U_{n+1,2} x_{n}-U_{n, 2} x_{n}\right\| \\
& =\mu_{1}\left\|\mu_{2} T_{2} U_{n+1,3} x_{n}-\mu_{2} T_{2} U_{n, 3} x_{n}\right\| \\
& \leq \mu_{1} \mu_{2}\left\|U_{n+1,3} x_{n}-U_{n, 3} x_{n}\right\| \\
& \leq \ldots \\
& \leq \mu_{1} \mu_{2} \ldots \mu_{n}\left\|U_{n+1, n+1} x_{n}-U_{n, n+1} x_{n}\right\| \\
& \leq M_{1} \prod_{i=1}^{n} \mu_{i}
\end{aligned}
$$

where $M_{1} \geq 0$ is an appropriate constant such that $\left\|U_{n+1, n+1} x_{n}-U_{n, n+1} x_{n}\right\| \leq M_{1}$ for all $n \geq 0$. From $I-\alpha_{n} B$ is nonexpansive, we have

$$
\begin{aligned}
\left\|v_{n+1}-v_{n}\right\| & =\left\|P_{C}\left(x_{n+1}-\alpha_{n+1} B x_{n+1}\right)-P_{C}\left(x_{n}-\alpha_{n} B x_{n}\right)\right\| \\
& \leq\left\|\left(x_{n+1}-\alpha_{n+1} B x_{n+1}\right)-\left(x_{n}-\alpha_{n} B x_{n}\right)\right\| \\
& \leq\left\|\left(x_{n+1}-\alpha_{n+1} B x_{n+1}\right)-\left(x_{n}-\alpha_{n+1} B x_{n}\right)\right\|+\left|\alpha_{n+1}-\alpha_{n}\right|\left\|B x_{n}\right\| \\
& \leq\left\|x_{n+1}-x_{n}\right\|+\left|\alpha_{n+1}-\alpha_{n}\right|\left\|B x_{n}\right\| .
\end{aligned}
$$

From (3.3) and (3.15), we have

$$
\begin{aligned}
\left\|y_{n+1}-y_{n}\right\|= & \left\|\left(1-\delta_{n+1}\right)\left(x_{n+1}-x_{n}\right)+\delta_{n+1}\left(v_{n+1}-v_{n}\right)+\left(\delta_{n+1}-\delta_{n}\right)\left(v_{n}-x_{n}\right)\right\| \\
\leq & \left(1-\delta_{n+1}\right)\left\|x_{n+1}-x_{n}\right\|+\delta_{n+1}\left\|v_{n+1}-v_{n}\right\|+\left|\delta_{n+1}-\delta_{n}\right|\left\|v_{n}-x_{n}\right\| \\
\leq & \left(1-\delta_{n+1}\right)\left\|x_{n+1}-x_{n}\right\|+\delta_{n+1}\left\{\left\|x_{n+1}-x_{n}\right\|+\left|\alpha_{n+1}-\alpha_{n}\right|\left\|B x_{n}\right\|\right\}(3.1) \\
& +\left|\delta_{n}-\delta_{n+1}\right|\left\|x_{n}-v_{n}\right\| \\
= & \left\|x_{n+1}-x_{n}\right\|+\delta_{n+1}\left|\alpha_{n+1}-\alpha_{n}\right|\left\|B x_{n}\right\|+\left|\delta_{n}-\delta_{n+1}\right|\left\|x_{n}-v_{n}\right\| .
\end{aligned}
$$


Now, we compute $\left\|u_{n+1}-u_{n}\right\|$ and $\left\|e_{n+1}-e_{n}\right\|$. Consider the following computation:

$$
\begin{aligned}
\left\|u_{n+1}-u_{n}\right\|= & \left\|\Im_{n+1}^{M} y_{n+1}-\Im_{n}^{M} y_{n}\right\| \\
\leq & \left\|\Im_{n+1}^{M} y_{n+1}-\Im_{n+1}^{M} y_{n}\right\|+\left\|\Im_{n+1}^{M} y_{n}-\Im_{n}^{M} y_{n}\right\| \\
\leq & \left\|y_{n+1}-y_{n}\right\|+\left\|\Im_{n+1}^{M} y_{n}-\Im_{n}^{M} y_{n}\right\| \\
\leq & \left\|x_{n+1}-x_{n}\right\|+\delta_{n+1}\left|\alpha_{n+1}-\alpha_{n}\right|\left\|B x_{n}\right\|+\left|\delta_{n}-\delta_{n+1}\right|\left\|x_{n}-v_{n}\right\| \\
& +\left\|\Im_{n+1}^{M} y_{n}-\Im_{n}^{M} y_{n}\right\|
\end{aligned}
$$

and

$$
\begin{aligned}
\left\|e_{n+1}-e_{n}\right\|= & \left\|p_{C}\left(W_{n+1} u_{n+1}-\lambda_{n+1} B W_{n+1} u_{n+1}\right)-P_{C}\left(W_{n} u_{n}-\lambda_{n} B W_{n} u_{n}\right)\right\| \\
\leq & \left\|\left(W_{n+1} u_{n+1}-\lambda_{n+1} B W_{n+1} u_{n+1}\right)-\left(W_{n} u_{n}-\lambda_{n} B W_{n} u_{n}\right)\right\| \\
= & \|\left(W_{n+1} u_{n+1}-\lambda_{n+1} B W_{n+1} u_{n+1}\right)-\left(W_{n+1} u_{n}-\lambda_{n+1} B W_{n+1} u_{n}\right) \\
& +\left(W_{n+1} u_{n}-\lambda_{n+1} B W_{n+1} u_{n}\right)-\left(W_{n} u_{n}-\lambda_{n} B W_{n} u_{n}\right) \| \\
\leq & \left\|\left(W_{n+1} u_{n+1}-\lambda_{n+1} B W_{n+1} u_{n+1}\right)-\left(W_{n+1} u_{n}-\lambda_{n+1} B W_{n+1} u_{n}\right)\right\| \\
& +\left\|W_{n+1} u_{n}-W_{n} u_{n}\right\|+\left\|\lambda_{n} B W_{n} u_{n}-\lambda_{n+1} B W_{n+1} u_{n}\right\| \\
\leq & \left\|u_{n+1}-u_{n}\right\|+M_{1} \prod_{i=1}^{n} \mu_{i}+\lambda_{n}\left\|B W_{n} u_{n}\right\|+\lambda_{n+1}\left\|B W_{n+1} u_{n}\right\| \\
\leq & \left\|x_{n+1}-x_{n}\right\|+\delta_{n+1}\left|\alpha_{n+1}-\alpha_{n}\right|\left\|B x_{n}\right\|+\left|\delta_{n}-\delta_{n+1}\right|\left\|x_{n}-v_{n}\right\| \\
& +\left\|\Im_{n+1}^{M} y_{n}-\Im_{n}^{M} y_{n}\right\|+M_{1} \prod_{i=1}^{n} \mu_{i}+\lambda_{n}\left\|B W_{n} u_{n}\right\|+\lambda_{n+1}\left\|B W_{n+1} u_{n}\right\| .
\end{aligned}
$$

Setting

$$
l_{n}=\frac{x_{n+1}-\beta_{n} x_{n}}{1-\beta_{n}}=\frac{\varepsilon_{n} \gamma f\left(u_{n}\right)+\left(\left(1-\beta_{n}\right) I-\varepsilon_{n} A\right) e_{n}}{1-\beta_{n}},
$$

we have $x_{n+1}=\left(1-\beta_{n}\right) l_{n}+\beta_{n} x_{n}, n \geq 1$. It follows that

$$
\begin{aligned}
l_{n+1}-l_{n}= & \frac{\varepsilon_{n+1} \gamma f\left(u_{n+1}\right)+\left(\left(1-\beta_{n+1}\right) I-\varepsilon_{n+1} A\right) e_{n+1}}{1-\beta_{n+1}} \\
& -\frac{\varepsilon_{n} \gamma f\left(u_{n}\right)+\left(\left(1-\beta_{n}\right) I-\varepsilon_{n} A\right) e_{n}}{1-\beta_{n}} \\
= & \frac{\varepsilon_{n+1}}{1-\beta_{n+1}}\left(\gamma f\left(u_{n+1}\right)-A e_{n+1}\right)+\frac{\varepsilon_{n}}{1-\beta_{n}}\left(A e_{n}-\gamma f\left(u_{n}\right)\right)+\left(e_{n+1}-e_{n}\right) .
\end{aligned}
$$

It follows from (3.18) and (3.19) that

$$
\begin{aligned}
\left\|l_{n+1}-l_{n}\right\|-\left\|x_{n+1}-x_{n}\right\| \leq & \frac{\varepsilon_{n+1}}{1-\beta_{n+1}}\left\|\gamma f\left(u_{n+1}\right)-A e_{n+1}\right\|+\frac{\varepsilon_{n}}{1-\beta_{n}}\left\|A e_{n}-\gamma f\left(u_{n}\right)\right\| \\
& +\delta_{n+1}\left|\alpha_{n+1}-\alpha_{n}\right|\left\|B x_{n}\right\|+\left|\delta_{n}-\delta_{n+1}\right|\left\|x_{n}-v_{n}\right\| \\
& +\left\|\Im_{n+1}^{M} y_{n}-\Im_{n}^{M} y_{n}\right\|+M_{1} \prod_{i=1}^{n} \mu_{i}+\lambda_{n}\left\|B W_{n} u_{n}\right\| \\
& +\lambda_{n+1}\left\|B W_{n+1} u_{n}\right\| \\
\leq & \frac{\varepsilon_{n+1}}{1-\beta_{n+1}}\left(\left\|\gamma f\left(u_{n+1}\right)\right\|+\left\|A e_{n+1}\right\|\right)+\frac{\varepsilon_{n}}{1-\beta_{n}}\left(\left\|A e_{n}\right\|+\left\|\gamma f\left(u_{n}\right)\right\|\right) \\
& +\delta_{n+1}\left|\alpha_{n+1}-\alpha_{n}\right|\left\|B x_{n}\right\|+\left|\delta_{n}-\delta_{n+1}\right|\left\|x_{n}-v_{n}\right\| \\
& +\left\|\Im_{n+1}^{M} y_{n}-\Im_{n}^{M} y_{n}\right\|+M_{1} \prod_{i=1}^{n} \mu_{i}+\lambda_{n}\left\|B W_{n} u_{n}\right\| \\
& +\lambda_{n+1}\left\|B W_{n+1} u_{n}\right\| .
\end{aligned}
$$


This together with conditions (C1)-(C4) and (3.11) imply that

$$
\limsup _{n \rightarrow \infty}\left(\left\|l_{n+1}-l_{n}\right\|-\left\|x_{n+1}-x_{n}\right\|\right) \leq 0 .
$$

By Lemma 2.8, we obtain

$$
\lim _{n \rightarrow \infty}\left\|l_{n}-x_{n}\right\|=0 \text {. }
$$

Consequently,

$$
\lim _{n \rightarrow \infty}\left\|x_{n+1}-x_{n}\right\|=\lim _{n \rightarrow \infty}\left(1-\beta_{n}\right)\left\|l_{n}-x_{n}\right\|=0 .
$$

Applying (3.11), (3.21) and conditions (C3), (C4) to (3.15) and (3.17), we obtain that

$$
\lim _{n \rightarrow \infty}\left\|u_{n+1}-u_{n}\right\|=\lim _{n \rightarrow \infty}\left\|v_{n+1}-v_{n}\right\|=0 .
$$

Step 5. We show that $\lim _{n \rightarrow \infty}\left\|W_{n} e_{n}-e_{n}\right\|=0$.

For any $\tilde{x} \in \Theta$ and (3.5), we obtain

$$
\begin{aligned}
\left\|v_{n}-\tilde{x}\right\|^{2} & =\left\|P_{C}\left(x_{n}-\alpha_{n} B x_{n}\right)-P_{C}\left(\tilde{x}-\alpha_{n} B \tilde{x}\right)\right\|^{2} \\
& \leq\left\|\left(x_{n}-\alpha_{n} B x_{n}\right)-\left(\tilde{x}-\alpha_{n} B \tilde{x}\right)\right\|^{2} \\
& \leq\left\|x_{n}-\tilde{x}\right\|^{2}+\left(\alpha_{n}^{2}-2 \alpha_{n} \xi\right)\left\|B x_{n}-B \tilde{x}\right\|^{2} .
\end{aligned}
$$

By Lemma 2.2(iv) and (3.23), we have

$$
\begin{aligned}
\left\|y_{n}-\tilde{x}\right\|^{2} & \leq\left(1-\delta_{n}\right)\left\|x_{n}-\tilde{x}\right\|^{2}+\delta_{n}\left\|v_{n}-\tilde{x}\right\|^{2} \\
& \leq\left(1-\delta_{n}\right)\left\|x_{n}-\tilde{x}\right\|^{2}+\delta_{n}\left\{\left\|x_{n}-\tilde{x}\right\|^{2}+\left(\alpha_{n}^{2}-2 \alpha_{n} \xi\right)\left\|B x_{n}-B \tilde{x}\right\|^{2}\right\} \\
& =\left\|x_{n}-\tilde{x}\right\|^{2}+\left(\alpha_{n}^{2}-2 \alpha_{n} \xi\right) \delta_{n}\left\|B x_{n}-B \tilde{x}\right\| .^{2}
\end{aligned}
$$

So, from (3.8) and (3.24), we derive

$$
\left\|e_{n}-\tilde{x}\right\|^{2} \leq\left\|u_{n}-\tilde{x}\right\|^{2} \leq\left\|y_{n}-\tilde{x}\right\|^{2} \leq\left\|x_{n}-\tilde{x}\right\|^{2}+\left(\alpha_{n}^{2}-2 \alpha_{n} \xi\right) \delta_{n}\left\|B x_{n}-B \tilde{x}\right\| .^{2}
$$

From (3.3), we have

$$
\begin{aligned}
\left\|x_{n+1}-\tilde{x}\right\|^{2}= & \left\|\left(\left(1-\beta_{n}\right) I-\varepsilon_{n} A\right)\left(e_{n}-\tilde{x}\right)+\beta_{n}\left(x_{n}-\tilde{x}\right)+\varepsilon_{n}\left(\gamma f\left(u_{n}\right)-A \tilde{x}\right)\right\|^{2} \\
= & \left\|\left(\left(1-\beta_{n}\right) I-\varepsilon_{n} A\right)\left(e_{n}-\tilde{x}\right)+\beta_{n}\left(x_{n}-\tilde{x}\right)\right\|^{2} \\
& +\varepsilon_{n}^{2}\left\|\gamma f\left(u_{n}\right)-A \tilde{x}\right\|^{2}+2 \beta_{n} \varepsilon_{n}\left\langle x_{n}-\tilde{x}, \gamma f\left(u_{n}\right)-A \tilde{x}\right\rangle \\
& +2 \varepsilon_{n}\left\langle\left(\left(1-\beta_{n}\right) I-\varepsilon_{n} A\right)\left(e_{n}-\tilde{x}\right), \gamma f\left(u_{n}\right)-A \tilde{x}\right\rangle \\
\leq & \left(\left(1-\beta_{n}-\varepsilon_{n} \bar{\gamma}\right)\left\|e_{n}-\tilde{x}\right\|+\beta_{n}\left\|x_{n}-\tilde{x}\right\|\right)^{2}+\varepsilon_{n} L_{n} \\
\leq & \left(1-\beta_{n}-\varepsilon_{n} \bar{\gamma}\right)^{2}\left\|e_{n}-\tilde{x}\right\|^{2}+\beta_{n}^{2}\left\|x_{n}-\tilde{x}\right\|^{2} \\
& +2\left(1-\beta_{n}-\varepsilon_{n} \bar{\gamma}\right) \beta_{n}\left\|e_{n}-\tilde{x}\right\|\left\|x_{n}-\tilde{x}\right\|+\varepsilon_{n} L_{n} \\
\leq & \left.\left(1-\varepsilon_{n} \bar{\gamma}\right)^{2}-2\left(1-\varepsilon_{n} \bar{\gamma}\right) \beta_{n}+\beta_{n}^{2}\right]\left\|e_{n}-\tilde{x}\right\|^{2} \\
& +\left(1-\beta_{n}-\varepsilon_{n} \bar{\gamma}\right) \beta_{n}\left\{\left\|e_{n}-\tilde{x}\right\|^{2}+\left\|x_{n}-\tilde{x}\right\|^{2}\right\}+\beta_{n}^{2}\left\|x_{n}-\tilde{x}\right\|^{2}+\varepsilon_{n} L_{n} \\
= & \left(1-\varepsilon_{n} \bar{\gamma}\right)\left(1-\beta_{n}-\varepsilon_{n} \bar{\gamma}\right)\left\|e_{n}-\tilde{x}\right\|^{2}+\left(1-\varepsilon_{n} \bar{\gamma}\right) \beta_{n}\left\|x_{n}-\tilde{x}\right\|^{2}+\varepsilon_{n} L_{n} \\
\leq & \left(1-\varepsilon_{n} \bar{\gamma}\right)\left(1-\beta_{n}-\varepsilon_{n} \bar{\gamma}\right)\left\{\left\|x_{n}-\tilde{x}\right\|^{2}+\left(\alpha_{n}^{2}-2 \alpha_{n} \xi\right) \delta_{n}\left\|B x_{n}-B \tilde{x}\right\|^{2}\right\} \\
& +\left(1-\varepsilon_{n} \bar{\gamma}\right) \beta_{n}\left\|x_{n}-\tilde{x}\right\|^{2}+\varepsilon_{n} L_{n} \\
= & \left(1-\varepsilon_{n} \bar{\gamma}\right)^{2}\left\|x_{n}-\tilde{x}\right\|^{2} \\
& +\left(1-\varepsilon_{n} \bar{\gamma}\right)\left(1-\beta_{n}-\varepsilon_{n} \bar{\gamma}\right)\left(\alpha_{n}^{2}-2 \alpha_{n} \xi\right) \delta_{n}\left\|B x_{n}-B \tilde{x}\right\|^{2}+\varepsilon_{n} L_{n} \\
\leq & \left\|x_{n}-\tilde{x}\right\|^{2}+\left(1-\varepsilon_{n} \bar{\gamma}\right)\left(1-\beta_{n}-\varepsilon_{n} \bar{\gamma}\right)\left(\alpha_{n}^{2}-2 \alpha_{n} \xi\right) \delta_{n}\left\|B x_{n}-B \tilde{x}\right\|^{2}+\varepsilon_{n} L_{n} .
\end{aligned}
$$


It follows that

$$
\begin{aligned}
& \left(1-\varepsilon_{n} \bar{\gamma}\right)\left(1-\beta_{n}-\varepsilon_{n} \bar{\gamma}\right)\left(2 g \xi-e^{2}\right) b\left\|B x_{n}-B \tilde{x}\right\|^{2} \\
\leq & \left(1-\varepsilon_{n} \bar{\gamma}\right)\left(1-\beta_{n}-\varepsilon_{n} \bar{\gamma}\right)\left(2 \alpha_{n} \xi-\alpha_{n}^{2}\right) \delta_{n}\left\|B x_{n}-B \tilde{x}\right\|^{2} \\
\leq & \left\|x_{n}-\tilde{x}\right\|^{2}-\left\|x_{n+1}-\tilde{x}\right\|^{2}+\varepsilon_{n} L_{n} \\
\leq & \left\|x_{n}-x_{n+1}\right\|\left(\left\|x_{n}-\tilde{x}\right\|+\left\|x_{n+1}-\tilde{x}\right\|\right)+\varepsilon_{n} L_{n},
\end{aligned}
$$

where

$$
\begin{aligned}
L_{n}= & \varepsilon_{n}\left\|\gamma f\left(u_{n}\right)-A \tilde{x}\right\|^{2}+2 \beta_{n}\left\langle x_{n}-\tilde{x}, \gamma f\left(u_{n}\right)-A \tilde{x}\right\rangle \\
& +2\left\langle\left(\left(1-\beta_{n}\right) I-\varepsilon_{n} A\right)\left(e_{n}-\tilde{x}\right), \gamma f\left(u_{n}\right)-A \tilde{x}\right\rangle .
\end{aligned}
$$

By conditions (C1), (C2) and (3.21), we obtain

$$
\lim _{n \rightarrow \infty}\left\|B x_{n}-B \tilde{x}\right\|=0 .
$$

Since $P_{C}$ is firmly nonexpansive mapping, we have

$$
\begin{aligned}
\left\|v_{n}-\tilde{x}\right\|^{2}= & \left\|P_{C}\left(x_{n}-\alpha_{n} B x_{n}\right)-P_{C}\left(\tilde{x}-\alpha_{n} B \tilde{x}\right)\right\|^{2} \\
\leq & \left\langle\left(x_{n}-\alpha_{n} B x_{n}\right)-\left(\tilde{x}-\alpha_{n} B \tilde{x}\right), v_{n}-\tilde{x}\right\rangle \\
= & \frac{1}{2}\left\{\left\|\left(x_{n}-\alpha_{n} B x_{n}\right)-\left(\tilde{x}-\alpha_{n} B \tilde{x}\right)\right\|^{2}+\left\|v_{n}-\tilde{x}\right\|^{2}\right. \\
& \left.-\left\|\left(x_{n}-\alpha_{n} B x_{n}\right)-\left(\tilde{x}-\alpha_{n} B \tilde{x}\right)-\left(v_{n}-\tilde{x}\right)\right\|^{2}\right\} \\
\leq & \frac{1}{2}\left\{\left\|x_{n}-\tilde{x}\right\|^{2}+\left\|v_{n}-\tilde{x}\right\|^{2}-\left\|\left(x_{n}-v_{n}\right)-\alpha_{n}\left(B x_{n}-B \tilde{x}\right)\right\|^{2}\right\} \\
\leq & \frac{1}{2}\left\{\left\|x_{n}-\tilde{x}\right\|^{2}+\left\|v_{n}-\tilde{x}\right\|^{2}-\left\|x_{n}-v_{n}\right\|^{2}\right. \\
& \left.-\alpha_{n}^{2}\left\|B x_{n}-B \tilde{x}\right\|^{2}+2 \alpha_{n}\left\|x_{n}-v_{n}\right\|\left\|B x_{n}-B \tilde{x}\right\|\right\} .
\end{aligned}
$$

Hence, we have

$$
\left\|v_{n}-\tilde{x}\right\|^{2} \leq\left\|x_{n}-\tilde{x}\right\|^{2}-\left\|x_{n}-v_{n}\right\|^{2}+2 \alpha_{n}\left\|x_{n}-v_{n}\right\|\left\|B x_{n}-B \tilde{x}\right\|
$$

and so

$$
\begin{aligned}
\left\|y_{n}-\tilde{x}\right\|^{2} & \leq\left(1-\delta_{n}\right)\left\|x_{n}-\tilde{x}\right\|^{2}+\delta_{n}\left\|v_{n}-\tilde{x}\right\|^{2} \\
& \leq\left(1-\delta_{n}\right)\left\|x_{n}-\tilde{x}\right\|^{2}+\delta_{n}\left\{\left\|x_{n}-\tilde{x}\right\|^{2}-\left\|x_{n}-v_{n}\right\|^{2}+2 \alpha_{n}\left\|x_{n}-v_{n}\right\|\left\|B x_{n}-B \tilde{x}\right\|\right\} \\
& =\left\|x_{n}-\tilde{x}\right\|^{2}-\delta_{n}\left\|x_{n}-v_{n}\right\|^{2}+2 \delta_{n} \alpha_{n}\left\|x_{n}-v_{n}\right\|\left\|B x_{n}-B \tilde{x}\right\| .
\end{aligned}
$$

Using (3.26) and (3.28), we also have

$$
\begin{aligned}
\left\|x_{n+1}-\tilde{x}\right\|^{2} \leq & \left(1-\varepsilon_{n} \bar{\gamma}\right)\left(1-\beta_{n}-\varepsilon_{n} \bar{\gamma}\right)\left\|e_{n}-\tilde{x}\right\|^{2}+\left(1-\varepsilon_{n} \bar{\gamma}\right) \beta_{n}\left\|x_{n}-\tilde{x}\right\|^{2}+\varepsilon_{n} L_{n} \\
\leq & \left(1-\varepsilon_{n} \bar{\gamma}\right)\left(1-\beta_{n}-\varepsilon_{n} \bar{\gamma}\right)\left\|u_{n}-\tilde{x}\right\|^{2}+\left(1-\varepsilon_{n} \bar{\gamma}\right) \beta_{n}\left\|x_{n}-\tilde{x}\right\|^{2}+\varepsilon_{n} L_{n} \\
\leq & \left(1-\varepsilon_{n} \bar{\gamma}\right)\left(1-\beta_{n}-\varepsilon_{n} \bar{\gamma}\right)\left\|y_{n}-\tilde{x}\right\|^{2}+\left(1-\varepsilon_{n} \bar{\gamma}\right) \beta_{n}\left\|x_{n}-\tilde{x}\right\|^{2}+\varepsilon_{n} L_{n} \\
\leq & \left(1-\varepsilon_{n} \bar{\gamma}\right)\left(1-\beta_{n}-\varepsilon_{n} \bar{\gamma}\right)\left\{\left\|x_{n}-\tilde{x}\right\|^{2}-\delta_{n}\left\|x_{n}-v_{n}\right\|^{2}+2 \delta_{n} \alpha_{n}\left\|x_{n}-v_{n}\right\|\left\|B x_{n}-B \tilde{x}\right\|\right\} \\
& +\left(1-\varepsilon_{n} \bar{\gamma}\right) \beta_{n}\left\|x_{n}-\tilde{x}\right\|^{2}+\varepsilon_{n} L_{n} \\
\leq & \left\|x_{n}-\tilde{x}\right\|^{2}-\left(1-\varepsilon_{n} \bar{\gamma}\right)\left(1-\beta_{n}-\varepsilon_{n} \bar{\gamma}\right) \delta_{n}\left\|x_{n}-v_{n}\right\|^{2} \\
& +2\left(1-\varepsilon_{n} \bar{\gamma}\right)\left(1-\beta_{n}-\varepsilon_{n} \bar{\gamma}\right) \delta_{n} \alpha_{n}\left\|x_{n}-v_{n}\right\|\left\|B x_{n}-B \tilde{x}\right\|+\varepsilon_{n} L_{n} .
\end{aligned}
$$


It follow that

$$
\begin{aligned}
& \left(1-\varepsilon_{n} \bar{\gamma}\right)\left(1-\beta_{n}-\varepsilon_{n} \bar{\gamma}\right) \delta_{n}\left\|x_{n}-v_{n}\right\|^{2} \\
\leq & \left\|x_{n}-x_{n+1}\right\|\left(\left\|x_{n}-\tilde{x}\right\|+\left\|x_{n+1}-\tilde{x}\right\|\right) \\
& +2\left(1-\varepsilon_{n} \bar{\gamma}\right)\left(1-\beta_{n}-\varepsilon_{n} \bar{\gamma}\right) \delta_{n} \alpha_{n}\left\|x_{n}-v_{n}\right\|\left\|B x_{n}-B \tilde{x}\right\|+\varepsilon_{n} L_{n} .
\end{aligned}
$$

From conditions (C1), C(4), (3.21) and (3.27), we obtain

$$
\lim _{n \rightarrow \infty}\left\|x_{n}-u_{n}\right\|=0
$$

Observe also that if $e_{n}=P_{C}\left(W_{n} u_{n}-\lambda_{n} B W_{n} u_{n}\right)$, then

$$
\begin{aligned}
\left\|e_{n}-\tilde{x}\right\|^{2} & =\left\|P_{C}\left(W_{n} u_{n}-\lambda_{n} B W_{n} u_{n}\right)-P_{C}\left(\tilde{x}-\lambda_{n} B \tilde{x}\right)\right\|^{2} \\
& \leq\left\|\left(W_{n} u_{n}-\lambda_{n} B W_{n} u_{n}\right)-\left(\tilde{x}-\lambda_{n} B \tilde{x}\right)\right\|^{2} \\
& =\left\|\left(W_{n} u_{n}-\lambda_{n} B W_{n} u_{n}\right)-\left(W_{n} \tilde{x}-\lambda_{n} B W_{n} \tilde{x}\right)\right\|^{2} \\
& \leq\left\|u_{n}-\tilde{x}\right\|^{2}+\left(\lambda_{n}^{2}-2 \lambda_{n} \xi\right)\left\|B W_{n} u_{n}-B \tilde{x}\right\|^{2} \\
& \leq\left\|x_{n}-\tilde{x}\right\|^{2}+\left(\lambda_{n}^{2}-2 \lambda_{n} \xi\right)\left\|B W_{n} u_{n}-B \tilde{x}\right\|^{2} .
\end{aligned}
$$

Substituting (3.30) in (3.26), we have

$$
\begin{aligned}
\left\|x_{n+1}-\tilde{x}\right\|^{2} \leq & \left(1-\varepsilon_{n} \bar{\gamma}\right)\left(1-\beta_{n}-\varepsilon_{n} \bar{\gamma}\right)\left\|e_{n}-\tilde{x}\right\|^{2}+\left(1-\varepsilon_{n} \bar{\gamma}\right) \beta_{n}\left\|x_{n}-\tilde{x}\right\|^{2}+\varepsilon_{n} L_{n} \\
\leq & \left(1-\varepsilon_{n} \bar{\gamma}\right)\left(1-\beta_{n}-\varepsilon_{n} \bar{\gamma}\right)\left\{\left\|x_{n}-\tilde{x}\right\|^{2}+\left(\lambda_{n}^{2}-2 \lambda_{n} \xi\right)\left\|B W_{n} u_{n}-B \tilde{x}\right\|^{2}\right\} \\
& +\left(1-\varepsilon_{n} \bar{\gamma}\right) \beta_{n}\left\|x_{n}-\tilde{x}\right\|^{2}+\varepsilon_{n} L_{n} \\
\leq & \left\|x_{n}-\tilde{x}\right\|^{2}+\left(1-\varepsilon_{n} \bar{\gamma}\right)\left(1-\beta_{n}-\varepsilon_{n} \bar{\gamma}\right)\left(\lambda_{n}^{2}-2 \lambda_{n} \xi\right)\left\|B W_{n} u_{n}-B \tilde{x}\right\|^{2}+\varepsilon_{n} L_{n} .
\end{aligned}
$$

It follows that

$$
\begin{aligned}
& \left(1-\varepsilon_{n} \bar{\gamma}\right)\left(1-\beta_{n}-\varepsilon_{n} \bar{\gamma}\right)\left(2 g \xi-e^{2}\right)\left\|B W_{n} u_{n}-B \tilde{x}\right\|^{2} \\
\leq & \left(1-\varepsilon_{n} \bar{\gamma}\right)\left(1-\beta_{n}-\varepsilon_{n} \bar{\gamma}\right)\left(2 \lambda_{n} \xi-\lambda_{n}^{2}\right)\left\|B W_{n} u_{n}-B \tilde{x}\right\|^{2} \\
\leq & \left\|x_{n}-x_{n+1}\right\|\left(\left\|x_{n}-\tilde{x}\right\|+\left\|x_{n+1}-\tilde{x}\right\|\right)+\varepsilon_{n} L_{n}
\end{aligned}
$$

Since $\left\|x_{n+1}-x_{n}\right\| \rightarrow 0(n \rightarrow \infty)$ and conditions $(\mathrm{C} 1)$ and $(\mathrm{C} 2)$, we obtain

$$
\lim _{n \rightarrow \infty}\left\|B W_{n} u_{n}-B \tilde{x}\right\|=0 .
$$

Since $P_{C}$ is firmly nonexpansive (Lemma 2.2 (iii)), we have

$$
\begin{aligned}
\left\|e_{n}-\tilde{x}\right\|^{2}= & \left\|P_{C}\left(W_{n} u_{n}-\lambda_{n} B W_{n} u_{n}\right)-P_{C}\left(\tilde{x}-\lambda_{n} B \tilde{x}\right)\right\|^{2} \\
\leq & \left\langle\left(W_{n} u_{n}-\lambda_{n} B W_{n} u_{n}\right)-\left(\tilde{x}-\lambda_{n} B \tilde{x}\right), e_{n}-\tilde{x}\right\rangle \\
= & \frac{1}{2}\left\{\left\|\left(W_{n} u_{n}-\lambda_{n} B W_{n} u_{n}\right)-\left(\tilde{x}-\lambda_{n} B \tilde{x}\right)\right\|^{2}+\left\|e_{n}-\tilde{x}\right\|^{2}\right. \\
& \left.-\left\|\left(W_{n} u_{n}-\lambda_{n} B W_{n} u_{n}\right)-\left(\tilde{x}-\lambda_{n} B \tilde{x}\right)-\left(e_{n}-\tilde{x}\right)\right\|^{2}\right\} \\
\leq & \frac{1}{2}\left\{\left\|u_{n}-\tilde{x}\right\|^{2}+\left\|e_{n}-\tilde{x}\right\|^{2}-\left\|\left(W_{n} u_{n}-e_{n}\right)-\lambda_{n}\left(B W_{n} u_{n}-B \tilde{x}\right)\right\|^{2}\right\} \\
\leq & \frac{1}{2}\left\{\left\|x_{n}-\tilde{x}\right\|^{2}+\left\|e_{n}-\tilde{x}\right\|^{2}-\left\|W_{n} u_{n}-e_{n}\right\|^{2}\right. \\
& \left.-\lambda_{n}^{2}\left\|B W_{n} u_{n}-B \tilde{x}\right\|^{2}+2 \lambda_{n}\left\|W_{n} u_{n}-e_{n}\right\|\left\|B W_{n} u_{n}-B \tilde{x}\right\|\right\} .
\end{aligned}
$$


Hence, we have

$$
\left\|e_{n}-\tilde{x}\right\|^{2} \leq\left\|x_{n}-\tilde{x}\right\|^{2}-\left\|W_{n} u_{n}-e_{n}\right\|^{2}+2 \lambda_{n}\left\|W_{n} u_{n}-e_{n}\right\|\left\|B W_{n} u_{n}-B \tilde{x}\right\| .
$$

Using (3.26) and (3.32), we also have

$$
\begin{aligned}
\left\|x_{n+1}-\tilde{x}\right\|^{2} \leq & \left(1-\varepsilon_{n} \bar{\gamma}\right)\left(1-\beta_{n}-\varepsilon_{n} \bar{\gamma}\right)\left\|e_{n}-\tilde{x}\right\|^{2}+\left(1-\varepsilon_{n} \bar{\gamma}\right) \beta_{n}\left\|x_{n}-\tilde{x}\right\|^{2}+\varepsilon_{n} L_{n} \\
\leq & \left(1-\varepsilon_{n} \bar{\gamma}\right)\left(1-\beta_{n}-\varepsilon_{n} \bar{\gamma}\right)\left\{\left\|x_{n}-\tilde{x}\right\|^{2}-\left\|W_{n} u_{n}-e_{n}\right\|^{2}\right. \\
& \left.+2 \lambda_{n}\left\|W_{n} u_{n}-e_{n}\right\|\left\|B W_{n} u_{n}-B \tilde{x}\right\|\right\}+\left(1-\varepsilon_{n} \bar{\gamma}\right) \beta_{n}\left\|x_{n}-\tilde{x}\right\|^{2}+\varepsilon_{n} L_{n} \\
\leq & \left\|x_{n}-\tilde{x}\right\|^{2}-\left(1-\varepsilon_{n} \bar{\gamma}\right)\left(1-\beta_{n}-\varepsilon_{n} \bar{\gamma}\right)\left\|W_{n} u_{n}-e_{n}\right\|^{2} \\
& +2\left(1-\varepsilon_{n} \bar{\gamma}\right)\left(1-\beta_{n}-\varepsilon_{n} \bar{\gamma}\right) \lambda_{n}\left\|W_{n} u_{n}-e_{n}\right\|\left\|B W_{n} u_{n}-B \tilde{x}\right\|+\varepsilon_{n} L_{n} .
\end{aligned}
$$

It follow that

$$
\begin{aligned}
& \left(1-\varepsilon_{n} \bar{\gamma}\right)\left(1-\beta_{n}-\varepsilon_{n} \bar{\gamma}\right)\left\|W_{n} u_{n}-e_{n}\right\|^{2} \\
\leq & \left\|x_{n}-x_{n+1}\right\|\left(\left\|x_{n}-\tilde{x}\right\|+\left\|x_{n+1}-\tilde{x}\right\|\right) \\
& +2\left(1-\varepsilon_{n} \bar{\gamma}\right)\left(1-\beta_{n}-\varepsilon_{n} \bar{\gamma}\right) \lambda_{n}\left\|W_{n} u_{n}-e_{n}\right\|\left\|B W_{n} u_{n}-B \tilde{x}\right\|+\varepsilon_{n} L_{n} .
\end{aligned}
$$

From conditions (C1), (3.21), and (3.31), we obtain

$$
\lim _{n \rightarrow \infty}\left\|W_{n} u_{n}-e_{n}\right\|=0 .
$$

For any $\tilde{x} \in \Theta$, note that $J_{r_{k, n}}^{F_{k}}$ is firmly nonexpansive (Lemma 2.7(2)) for $k \in\{1,2,3, \ldots$, $M$, then we have

$$
\begin{aligned}
\left\|\Im_{\Im_{n}}^{k} y_{n}-\tilde{x}\right\|^{2} & =\left\|J_{r_{k, n}}^{F_{n}} \Im_{n}^{k-1} y_{n}-J_{r_{k, n}}^{F_{k}} \tilde{x}\right\|^{2} \\
& \leq\left\langle J_{r_{k, n}}^{F_{n}} \Im_{n}^{k-1} y_{n}-J_{r_{k, n}}^{F_{k}} \tilde{x}, \Im_{n}^{k-1} y_{n}-\tilde{x}\right\rangle \\
& =\left\langle\Im_{\Im_{n}}^{k} y_{n}-\tilde{x}, \Im_{n}^{k-1} y_{n}-\tilde{x}\right\rangle \\
& =\frac{1}{2}\left(\left\|\Im_{n}^{k} y_{n}-\tilde{x}\right\|^{2}+\left\|\Im_{\Im_{n}^{k}}^{k-1} y_{n}-\tilde{x}\right\|^{2}-\left\|\Im_{\Im_{n}}^{k} y_{n}-\Im_{\Im_{n}}^{k-1} y_{n}\right\|^{2}\right) .
\end{aligned}
$$

So, we obtain

$$
\left\|\Im_{\Im_{n}^{k}}^{k} y_{n}-\tilde{x}\right\|^{2} \leq\left\|\Im_{n}^{k-1} y_{n}-\tilde{x}\right\|^{2}-\left\|\Im_{n}^{k} y_{n}-\Im_{n}^{k-1} y_{n}\right\|^{2}, \quad k=1,2,3, \ldots, M
$$

which implies that for each $k \in\{1,2,3, \ldots, M-1\}$,

$$
\begin{aligned}
\left\|\Im_{n}^{k} y_{n}-\tilde{x}\right\|^{2} \leq & \left\|\Im_{n}^{0} y_{n}-\tilde{x}\right\|^{2}-\left\|\Im_{\Im_{n}}^{k} y_{n}-\Im_{n}^{k-1} y_{n}\right\|^{2} \\
& -\left\|\Im_{n}^{k-1} y_{n}-\Im_{n}^{k-2} y_{n}\right\|^{2}-\cdots-\left\|\Im_{n}^{2} y_{n}-\Im_{n}^{1} y_{n}\right\|^{2}-\left\|\Im_{n}^{1} y_{n}-\Im_{n}^{0} y_{n}\right\|^{2} \\
\leq & \left\|y_{n}-\tilde{x}\right\|^{2}-\left\|\Im_{\Im_{n}}^{k} y_{n}-\Im_{n}^{k-1} y_{n}\right\|^{2} \\
\leq & \left\|x_{n}-\tilde{x}\right\|^{2}-\left\|\Im_{\Im_{n}}^{k} y_{n}-\Im_{n}^{k-1} y_{n}\right\|^{2} .
\end{aligned}
$$


Consequently, from (3.26) we derive that

$$
\begin{aligned}
\left\|x_{n+1}-\tilde{x}\right\|^{2} \leq & \left(1-\varepsilon_{n} \bar{\gamma}\right)\left(1-\beta_{n}-\varepsilon_{n} \bar{\gamma}\right)\left\|e_{n}-\tilde{x}\right\|^{2}+\left(1-\varepsilon_{n} \bar{\gamma}\right) \beta_{n}\left\|x_{n}-\tilde{x}\right\|^{2}+\varepsilon_{n} L_{n} \\
\leq & \left(1-\varepsilon_{n} \bar{\gamma}\right)\left(1-\beta_{n}-\varepsilon_{n} \bar{\gamma}\right)\left\|u_{n}-\tilde{x}\right\|^{2}+\left(1-\varepsilon_{n} \bar{\gamma}\right) \beta_{n}\left\|x_{n}-\tilde{x}\right\|^{2}+\varepsilon_{n} L_{n} \\
= & \left(1-\varepsilon_{n} \bar{\gamma}\right)\left(1-\beta_{n}-\varepsilon_{n} \bar{\gamma}\right)\left\|\Im_{n}^{k} y_{n}-\tilde{x}\right\|^{2}+\left(1-\varepsilon_{n} \bar{\gamma}\right) \beta_{n}\left\|x_{n}-\tilde{x}\right\|^{2}+\varepsilon_{n} L_{n} \\
\leq & \left(1-\varepsilon_{n} \bar{\gamma}\right)\left(1-\beta_{n}-\varepsilon_{n} \bar{\gamma}\right)\left\{\left\|x_{n}-\tilde{x}\right\|^{2}-\left\|\Im_{\Im_{n}}^{k} y_{n}-\Im_{n}^{k-1} y_{n}\right\|^{2}\right\} \\
& +\left(1-\varepsilon_{n} \bar{\gamma}\right) \beta_{n}\left\|x_{n}-\tilde{x}\right\|^{2}+\varepsilon_{n} L_{n} \\
\leq & \left\|x_{n}-\tilde{x}\right\|^{2}-\left(1-\varepsilon_{n} \bar{\gamma}\right)\left(1-\beta_{n}-\varepsilon_{n} \bar{\gamma}\right)\left\|\Im_{\Im_{n}}^{k} y_{n}-\Im_{n}^{k-1} y_{n}\right\|^{2}+\varepsilon_{n} L_{n} .
\end{aligned}
$$

Thus, we have

$$
\left(1-\varepsilon_{n} \bar{\gamma}\right)\left(1-\beta_{n}-\varepsilon_{n} \bar{\gamma}\right)\left\|\Im_{n}^{k} y_{n}-\Im_{n}^{k-1} y_{n}\right\|^{2} \leq\left\|x_{n}-x_{n+1}\right\|\left(\left\|x_{n}-\tilde{x}\right\|+\left\|x_{n+1}-\tilde{x}\right\|\right)+\varepsilon_{n} L_{n} .
$$

By $\liminf _{n \rightarrow \infty} \beta_{n}>0, \varepsilon_{n} \rightarrow 0$ as $n \rightarrow \infty$ and (3.21), so we deduce that

$$
\lim _{n \rightarrow \infty}\left\|\Im_{n}^{k} y_{n}-\Im_{n}^{k-1} y_{n}\right\|=0, \quad k=1,2, \ldots, M-1,
$$

that is,

$$
\left\|u_{n}^{(k)}-u_{n}^{(k-1)}\right\| \rightarrow 0 \text { as } n \rightarrow \infty .
$$

Therefore, we have

$$
\begin{aligned}
\left\|y_{n}-u_{n}\right\| & =\left\|\Im_{n}^{0} y_{n}-\Im_{n}^{k} y_{n}\right\| \\
& \leq\left\|\Im_{n}^{0} y_{n}-\Im_{n}^{1} y_{n}\right\|+\left\|\Im_{n}^{1} y_{n}-\Im_{n}^{2} y_{n}\right\|+\cdots+\left\|\Im_{n}^{M-1} y_{n}-\Im_{n}^{M} y_{n}\right\| .
\end{aligned}
$$

From (3.34), we have

$$
\lim _{n \rightarrow \infty}\left\|y_{n}-u_{n}\right\|=0
$$

Since $x_{n+1}=\epsilon_{n} \gamma f\left(u_{n}\right)+\beta_{n} x_{n}+\left(\left(1-\beta_{n}\right) I-\epsilon_{n} A\right) e_{n}$, we have

$$
\begin{aligned}
\left\|x_{n}-e_{n}\right\| & \leq\left\|x_{n}-x_{n+1}\right\|+\left\|x_{n+1}-e_{n}\right\| \\
& =\left\|x_{n}-x_{n+1}\right\|+\left\|\varepsilon_{n} \gamma f\left(u_{n}\right)+\beta_{n} x_{n}+\left(\left(1-\beta_{n}\right) I-\varepsilon_{n} A\right) e_{n}-e_{n}\right\| \\
& =\left\|x_{n}-x_{n+1}\right\|+\left\|\varepsilon_{n}\left(\gamma f\left(u_{n}\right)-A e_{n}\right)+\beta_{n}\left(x_{n}-e_{n}\right)\right\| \\
& \leq\left\|x_{n}-x_{n+1}\right\|+\varepsilon_{n}\left(\left\|\gamma f\left(u_{n}\right)\right\|+\left\|A e_{n}\right\|\right)+\beta_{n}\left\|x_{n}-e_{n}\right\|,
\end{aligned}
$$

that is,

$$
\left\|x_{n}-e_{n}\right\| \leq \frac{1}{1-\beta_{n}}\left\|x_{n}-x_{n+1}\right\|+\frac{\varepsilon_{n}}{1-\beta_{n}}\left(\left\|\gamma f\left(u_{n}\right)\right\|+\left\|A e_{n}\right\|\right) .
$$

By conditions (C1), (C2), and (3.21) it follows that

$$
\lim _{n \rightarrow \infty}\left\|x_{n}-e_{n}\right\|=0 .
$$

On the other hand, from (3.3), we have

$$
\left\|y_{n}-x_{n}\right\|=\delta_{n}\left\|v_{n}-x_{n}\right\| \text {. }
$$


Since $\lim _{n \rightarrow \infty}\left\|x_{n}-v_{n}\right\|=0$, we get

$$
\lim _{n \rightarrow \infty}\left\|y_{n}-x_{n}\right\|=0
$$

We observe that

$$
\begin{aligned}
\left\|W_{n} e_{n}-e_{n}\right\| & \leq\left\|W_{n} e_{n}-W_{n} u_{n}\right\|+\left\|W_{n} u_{n}-e_{n}\right\| \\
& \leq\left\|e_{n}-x_{n}+x_{n}-y_{n}+y_{n}-u_{n}\right\|+\left\|W_{n} u_{n}-e_{n}\right\| \\
& \leq\left\|e_{n}-x_{n}\right\|+\left\|x_{n}-y_{n}\right\|+\left\|y_{n}-u_{n}\right\|+\left\|W_{n} u_{n}-e_{n}\right\| .
\end{aligned}
$$

Consequently, we obtain

$$
\lim _{n \rightarrow \infty}\left\|W_{n} e_{n}-e_{n}\right\|=0 .
$$

Let $W$ be the mapping defined by (3.2). Since $\left\{e_{n}\right\}$ is bounded, applying Lemma 3.1(4) and (3.38), we have

$$
\left\|W e_{n}-e_{n}\right\| \leq\left\|W e_{n}-W_{n} e_{n}\right\|+\left\|W_{n} e_{n}-e_{n}\right\| \rightarrow 0 \text { as } n \rightarrow \infty .
$$

Step 6. We show that $q \in \Theta$, where $\Theta:=\cap_{n=1}^{\infty} F\left(T_{n}\right) \cap\left(\cap_{k=1}^{M} \operatorname{SEP}\left(F_{k}\right)\right) \cap \operatorname{VI}(C, B)$.

Since $\left\{x_{n}\right\}$ is bounded, we see that there exits a subsequence $\left\{x_{n_{i}}\right\}$ of $\left\{x_{n}\right\}$ which converges weakly to $q$. It follows from (3.37) and (3.36) that $y_{n_{i}} \rightarrow q$ and $e_{n_{i}} \rightarrow q$. From (3.35), we obtain that $\Im_{n_{i}}^{k} y_{n_{i}} \rightarrow q$ for $k=1,2, \ldots, M$.

First, we show that $q \in \cap_{k=1}^{M} \operatorname{SEP}\left(F_{k}\right)$. Since $u_{n}=\Im_{\Im_{n}}^{k} y_{n}$ for $k=1,2,3, \ldots, M$, we also have

$$
F_{k}\left(\Im_{n}^{k} y_{n}, y\right)+\frac{1}{r_{n}}\left\langle y-\Im_{n}^{k} y_{n}, \Im_{n}^{k} y_{n}-\Im_{n}^{k-1} y_{n}\right\rangle \geq 0, \quad \forall y \in C .
$$

If follows from (A2) that,

$$
\frac{1}{r_{n}}\left\langle y-\Im_{n}^{k} y_{n}, \Im_{n}^{k} y_{n}-\Im_{n}^{k-1} y_{n}\right\rangle \geq-F_{k}\left(\Im_{n}^{k} y_{n}, y\right) \geq F_{k}\left(y, \Im_{n}^{k} y_{n}\right) .
$$

Replacing $n$ by $n_{i}$, we have

$$
\left\langle y-\Im_{n_{i}}^{k} y_{n_{i}}, \frac{\Im_{n_{i}}^{k} y_{n_{i}}-\Im_{n_{i}}^{k-1} y_{n_{i}}}{r_{n_{i}}}\right\rangle \geq F_{k}\left(\gamma, \Im_{n_{i}}^{k} y_{n_{i}}\right) .
$$

Since $\frac{\Im_{n_{i}}^{k} y_{n_{i}}-\Im_{n_{i}}^{k-1} y_{n_{i}}}{r_{n_{i}}} \rightarrow 0$ and $\Im_{n_{i}}^{k} y_{n_{i}} \rightarrow q$, it follows by (A4) that

$$
F_{k}(y, q) \leq 0 \quad \forall y \in C
$$

for each $k=1,2,3, \ldots, M$.

For $t$ with $0<t \leq 1$ and $y \in H$, let $y_{t}=t y+(1-t) q$. Since $y \in C$ and $q \in C$, we have $y_{t} \in C$ and hence $F_{k}\left(y_{t}, q\right) \leq 0$. So, from (A1) and (A4) we have

$$
0=F_{k}\left(y_{t}, y_{t}\right) \leq t F_{k}\left(y_{t}, y\right)+(1-t) F_{k}\left(y_{t}, q\right) \leq t F_{k}\left(y_{t}, \gamma\right)
$$

and hence $F_{k}\left(y_{t} y\right) \geq 0$. From (A3), we have $F_{k}(q, y) \geq 0$ for all $y \in C$ and hence $q \in$ $\operatorname{SEP}\left(F_{k}\right)$ for $k=1,2,3, \ldots, M$, that is, $q \in \cap_{k=1}^{M} \operatorname{SEP}\left(F_{k}\right)$.

Next, we show that $q \in \cap_{n=1}^{\infty} F\left(T_{n}\right)$. By Lemma 3.1(2), we have $F(W)=\cap_{n=1}^{\infty} F\left(T_{n}\right)$. Assume $q \notin F(W)$. Since $e_{n_{i}} \rightarrow q$ and $q \neq W q$, it follows by the Opial's condition (Lemma 2.3) that 


$$
\begin{aligned}
\liminf _{i \rightarrow \infty}\left\|e_{n_{i}}-q\right\| & <\liminf _{i \rightarrow \infty}\left\|e_{n_{i}}-W q\right\| \\
& \leq \liminf _{i \rightarrow \infty}\left\{\left\|e_{n_{i}}-W e_{n_{i}}\right\|+\left\|W e_{n_{i}}-W_{q}\right\|\right\} \\
& \leq \liminf _{i \rightarrow \infty}\left\|e_{n_{i}}-q\right\|
\end{aligned}
$$

which derives a contradiction. Thus, we have $q \in F(W)=\cap_{n=1}^{\infty} F\left(T_{n}\right)$.

Finally, now we prove that $q \in V I(C, B)$.

We define the maximal monotone operator

$$
Q q_{1}= \begin{cases}B q_{1}+N_{C} q_{1}, & q_{1} \in C, \\ \emptyset, & q_{1} \notin C .\end{cases}
$$

For any given $\left(q_{1}, q_{2}\right) \in G(Q)$, hence $q_{2}-B q_{1} \in N_{C} q_{1}$. Since $e_{n} \in C$ we see from the definition of $N_{C}$ that

$$
\left\langle q_{1}-e_{n}, q_{2}-B q_{1}\right\rangle \geq 0 .
$$

On the other hand, from $e_{n}=P_{C}\left(W_{n} u_{n}-\alpha_{n} B W_{n} u_{n}\right)$, we have

$$
\left\langle q_{1}-e_{n}, e_{n}-\left(W_{n} u_{n}-\alpha_{n} B W_{n} u_{n}\right)\right\rangle \geq 0,
$$

that is

$$
\left\langle q_{1}-e_{n}, \frac{e_{n}-W_{n} u_{n}}{\alpha_{n}}+B W_{n} u_{n}\right\rangle \geq 0 .
$$

Therefore, we obtain

$$
\begin{aligned}
\left\langle q_{1}-e_{n_{i}}, q_{2}\right\rangle \geq & \left\langle q_{1}-e_{n_{i}}, B q_{1}\right\rangle \\
\geq & \left\langle q_{1}-e_{n_{i}}, B q_{1}\right\rangle-\left\langle q_{1}-e_{n_{i}} \frac{e_{n_{i}}-W_{n} u_{n_{i}}}{\alpha_{n_{i}}}+B W_{n} u_{n_{i}}\right\rangle \\
= & \left\langle q_{1}-e_{n_{i}}, B q_{1}-B W_{n} u_{n_{i}}-\frac{e_{n_{i}}-W_{n} u_{n_{i}}}{\alpha_{n_{i}}}\right\rangle \\
= & \left\langle q_{1}-e_{n_{i}}, B q_{1}-B e_{n_{i}}\right\rangle+\left\langle q_{1}-e_{n_{i}}, B e_{n_{i}}-B W_{n} u_{n_{i}}\right\rangle \\
& -\left\langle q_{1}-e_{n_{i}}, \frac{e_{n_{i}}-W_{n} u_{n_{i}}}{\alpha_{n_{i}}}\right\rangle \\
\geq & \left\langle q_{1}-e_{n_{i}}, B e_{n_{i}}-B W_{n} u_{n_{i}}\right\rangle-\left\langle q_{1}-e_{n_{i}} \frac{e_{n_{i}}-W_{n} u_{n_{i}}}{\alpha_{n_{i}}}\right\rangle,
\end{aligned}
$$

Since $\left\|e_{n_{i}}-W_{n} u_{n_{i}}\right\| \rightarrow 0$ as $i \rightarrow \infty$ and $B$ is Lipschitz continuous we obtain that

$$
\left\langle q_{1}-q_{1} q_{2}\right\rangle \geq 0 \text {. }
$$

Notice that $Q$ is maximal monotone, we obtain that $q \in Q^{-1} 0$ and hence $q \in V I(C, B)$. This implies $q \in \Theta$. Since $z=P_{\Theta}(\gamma f+(I-A))(z)$, we have

$$
\limsup _{n \rightarrow \infty}\left\langle x_{n}-z, \gamma f(z)-A z\right\rangle=\lim _{i \rightarrow \infty}\left\langle x_{n_{i}}-z, \gamma f(z)-A z\right\rangle=\langle q-z, \gamma f(z)-A z\rangle \leq 0 .
$$

On the other hand, we have

$$
\begin{aligned}
\left\langle e_{n}-z, \gamma f(z)-A z\right\rangle & =\left\langle e_{n}-x_{n}, \gamma f(z)-A z\right\rangle+\left\langle x_{n}-z, \gamma f(z)-A z\right\rangle \\
& \leq\left\|e_{n}-x_{n}\right\|\|\gamma f(z)-A z\|+\left\langle x_{n}-z, \gamma f(z)-A z\right\rangle .
\end{aligned}
$$


From (3.36) and (3.41), we obtain that

$$
\limsup _{n \rightarrow \infty}\left\langle e_{n}-z, \gamma f(z)-A z\right\rangle \leq 0 .
$$

Step 7. Finally, we show that $\left\{x_{n}\right\}$ converges strongly to $z=P_{\Theta}(I-A+\gamma f)(z)$. Indeed, from (3.3), we have

$$
\begin{aligned}
& \left\|x_{n+1}-z\right\|^{2}=\left\|\left(\left(1-\beta_{n}\right) I-\varepsilon_{n} A\right)\left(e_{n}-z\right)+\beta_{n}\left(x_{n}-z\right)+\varepsilon_{n}\left(\gamma f\left(u_{n}\right)-A z\right)\right\|^{2} \\
& =\left\|\left(\left(1-\beta_{n}\right) I-\varepsilon_{n} A\right)\left(e_{n}-z\right)+\beta_{n}\left(x_{n}-z\right)\right\|^{2} \\
& +\varepsilon_{n}^{2}\left\|\gamma f\left(u_{n}\right)-A z\right\|^{2}+2 \beta_{n} \varepsilon_{n}\left\langle x_{n}-z, \gamma f\left(u_{n}\right)-A z\right\rangle \\
& +2 \varepsilon_{n}\left\langle\left(\left(1-\beta_{n}\right) I-\varepsilon_{n} A\right)\left(e_{n}-z\right), \gamma f\left(u_{n}\right)-A z\right\rangle \\
& \leq\left(\left(1-\beta_{n}-\varepsilon_{n} \bar{\gamma}\right)\left\|e_{n}-z\right\|+\beta_{n}\left\|x_{n}-z\right\|\right)^{2}+\varepsilon_{n}^{2}\left\|\gamma f\left(u_{n}\right)-A z\right\|^{2} \\
& +2 \beta_{n} \varepsilon_{n} \gamma\left\langle x_{n}-z_{1} f\left(u_{n}\right)-f(z)\right\rangle+2 \beta_{n} \varepsilon_{n}\left\langle x_{n}-z, \gamma f(z)-A z\right\rangle \\
& +2\left(1-\beta_{n}\right) \varepsilon_{n} \gamma\left\langle e_{n}-z_{1} f\left(u_{n}\right)-f(z)\right\rangle+2 \varepsilon_{n}\left\langle e_{n}-z_{,} \gamma f(z)-A z\right\rangle \\
& -2 \beta_{n} \varepsilon_{n}\left\langle e_{n}-z, \gamma f(z)-A z\right\rangle-2 \varepsilon_{n}^{2}\left\langle\left(A\left(e_{n}-z\right), \gamma f\left(u_{n}\right)-A z\right\rangle\right. \\
& \leq\left(1-\beta_{n}-\varepsilon_{n} \bar{\gamma}\right)^{2}\left\|e_{n}-z\right\|^{2}+\beta_{n}^{2}\left\|x_{n}-z\right\|^{2}+2\left(1-\beta_{n}-\varepsilon_{n} \bar{\gamma}\right) \beta_{n}\left\|e_{n}-z\right\|\left\|x_{n}-z\right\| \\
& +\varepsilon_{n}^{2}\left\|\gamma f\left(u_{n}\right)-A z\right\|^{2}+2 \beta_{n} \varepsilon_{n} \gamma\left\|x_{n}-z\right\|\left\|f\left(u_{n}\right)-f(z)\right\| \\
& +2\left(1-\beta_{n}\right) \varepsilon_{n} \gamma\left\|e_{n}-z\right\|\left\|f\left(u_{n}\right)-f(z)\right\|+2 \beta_{n} \varepsilon_{n}\left\|x_{n}-z\right\|\|\gamma f(z)-A z\| \\
& -2 \beta_{n} \varepsilon_{n}\left\|e_{n}-z\right\|\|\gamma f(z)-A z\|-2 \varepsilon_{n}^{2}\left\|A\left(e_{n}-z\right)\right\|\left\|\gamma f\left(u_{n}\right)-A z\right\| \\
& +2 \varepsilon_{n}\left\langle e_{n}-z, \gamma f(z)-A z\right\rangle \\
& \leq\left[\left(1-\varepsilon_{n} \bar{\gamma}\right)^{2}-2\left(1-\varepsilon_{n} \bar{\gamma}\right) \beta_{n}+\beta_{n}^{2}\right]\left\|e_{n}-z\right\|^{2}+\beta_{n}^{2}\left\|x_{n}-z\right\|^{2} \\
& +\left(1-\beta_{n}-\varepsilon_{n} \bar{\gamma}\right) \beta_{n}\left\{\left\|e_{n}-z\right\|^{2}+\left\|x_{n}-z\right\|^{2}\right\}+\varepsilon_{n}^{2}\left\|\gamma f\left(u_{n}\right)-A z\right\|^{2} \\
& +2 \beta_{n} \varepsilon_{n} \gamma \eta\left\|x_{n}-z\right\|\left\|u_{n}-z\right\|+2\left(1-\beta_{n}\right) \varepsilon_{n} \gamma \eta\left\|e_{n}-z\right\|\left\|u_{n}-z\right\| \\
& +2 \beta_{n} \varepsilon_{n}\left\|x_{n}-z\right\|\|\gamma f(z)-A z\|-2 \beta_{n} \varepsilon_{n}\left\|e_{n}-z\right\|\|\gamma f(z)-A z\| \\
& -2 \varepsilon_{n}^{2}\left\|A\left(e_{n}-z\right)\right\|\left\|\gamma f\left(u_{n}\right)-A z\right\|+2 \varepsilon_{n}\left\langle e_{n}-z, \gamma f(z)-A z\right\rangle \\
& \leq\left(1-\varepsilon_{n} \bar{\gamma}\right)\left(1-\beta_{n}-\varepsilon_{n} \bar{\gamma}\right)\left\|e_{n}-z\right\|^{2}+\left(1-\varepsilon_{n} \bar{\gamma}\right) \beta_{n}\left\|x_{n}-z\right\|^{2} \\
& +\varepsilon_{n}^{2}\left\|\gamma f\left(u_{n}\right)-A z\right\|^{2}+2 \beta_{n} \varepsilon_{n} \gamma \eta\left\|x_{n}-z\right\|^{2}+2\left(1-\beta_{n}\right) \varepsilon_{n} \gamma \eta\left\|x_{n}-z\right\|^{2} \\
& +2 \beta_{n} \varepsilon_{n}\left\|x_{n}-z\right\|\|\gamma f(z)-A z\|-2 \beta_{n} \varepsilon_{n}\left\|e_{n}-z\right\|\|\gamma f(z)-A z\| \\
& +2 \varepsilon_{n}^{2}\left\|A\left(e_{n}-z\right)\right\|\left\|\gamma f\left(u_{n}\right)-A z\right\|+2 \varepsilon_{n}\left\langle e_{n}-z, \gamma f(z)-A z\right\rangle \\
& \leq\left(1-\varepsilon_{n} \bar{\gamma}\right)\left(1-\beta_{n}-\varepsilon_{n} \bar{\gamma}\right)\left\|x_{n}-z\right\|^{2}+\left(1-\varepsilon_{n} \bar{\gamma}\right) \beta_{n}\left\|x_{n}-z\right\|^{2} \\
& +\varepsilon_{n}^{2}\left\|\gamma f\left(u_{n}\right)-A z\right\|^{2}+2 \varepsilon_{n} \gamma \eta\left\|x_{n}-z\right\|^{2} \\
& +2 \beta_{n} \varepsilon_{n}\left\|x_{n}-z\right\|\|\gamma f(z)-A z\|-2 \beta_{n} \varepsilon_{n}\left\|x_{n}-z\right\|\|\gamma f(z)-A z\| \\
& +2 \varepsilon_{n}^{2}\left\|A\left(e_{n}-z\right)\right\|\left\|\gamma f\left(u_{n}\right)-A z\right\|+2 \varepsilon_{n}\left\langle e_{n}-z, \gamma f(z)-A z\right\rangle \\
& =\left(1-2 \varepsilon_{n} \bar{\gamma}+\varepsilon_{n}^{2} \bar{\gamma}^{2}+2 \varepsilon_{n} \gamma \eta\right)\left\|x_{n}-z\right\|^{2}+\varepsilon_{n}^{2}\left\|\gamma f\left(u_{n}\right)-A z\right\|^{2} \\
& +2 \varepsilon_{n}^{2}\left\|A\left(e_{n}-z\right)\right\|\left\|\gamma f\left(u_{n}\right)-A z\right\|+2 \varepsilon_{n}\left\langle e_{n}-z, \gamma f(z)-A z\right\rangle \\
& =\left[1-2(\bar{\gamma}-\gamma \eta) \varepsilon_{n}\right]\left\|x_{n}-z\right\|^{2}+\varepsilon_{n}\left\{2\left\langle e_{n}-z, \gamma f(z)-A z\right\rangle+\varepsilon_{n} K\right\} \text {. }
\end{aligned}
$$

where $K$ is an appropriate constant such that

$$
K \geq \max \left\{\sup _{n \geq 1}\left\{\bar{\gamma}^{2}\left\|x_{n}-z\right\|^{2}+\left\|\gamma f\left(u_{n}\right)-A z\right\|^{2}+2\left\|A\left(e_{n}-z\right)\right\|\left\|\gamma f\left(u_{n}\right)-A z\right\|\right\}\right\},
$$

Set $b_{n}=2(\bar{\gamma}-\gamma \eta) \varepsilon_{n}$ and $c_{n}=\epsilon_{n}\left\{2\left\langle e_{n}-z, \gamma f(z)-A z\right\rangle+\epsilon_{n} K\right\}$. Then we have

$$
\left\|x_{n+1}-z\right\|^{2} \leq\left(1-b_{n}\right)\left\|x_{n}-z\right\|^{2}+c_{n}, \forall n \geq 0 .
$$

From the conditions (C1) and (3.42), we see that

$$
\lim _{n \rightarrow \infty} b_{n}=0, \quad \sum_{n=0}^{\infty} b_{n}=\infty \text { and } \limsup _{n \rightarrow \infty} c_{n} \leq 0 .
$$


Therefore, applying Lemma 2.8 to (3.43), we get that $\left\{x_{n}\right\}$ converges strongly to $z \in$ $\Theta$. This completes the proof.

Corollary 3.3. Let $C$ be a nonempty closed convex subset of a real Hilbert space $H$, let $F_{k} k \in\{1,2,3, \ldots, M\}$ be a bifunction from $C \times C$ to $\mathbb{R}$ satisfying (A1)-(A4) and let $B$ be $\xi$-inverse strongly monotone such that

$$
\Theta:=\left(\cap_{k=1}^{M} \operatorname{SEP}\left(F_{k}\right)\right) \cap \operatorname{VI}(C, B) \neq \emptyset .
$$

Let $f$ be a contraction of $H$ into itself with $\eta \in(0,1)$. Let $\left\{x_{n}\right\},\left\{y_{n}\right\}$ and $\left\{u_{n}\right\}$ be sequences generated by

$$
\left\{\begin{array}{l}
x_{1}=x \in C \text { chosen arbitrary, } \\
y_{n}=\left(1-\delta_{n}\right) x_{n}+\delta_{n} P_{C}\left(x_{n}-\alpha_{n} B x_{n}\right), \\
u_{n}=J_{r_{M}, n}^{F_{M}} F_{r_{M-1, n}} J_{r_{M-2, n}}^{F_{M-2}} \ldots J_{r_{2, n}, J_{r_{1, n}}}^{F_{1}} y_{n} \\
x_{n+1}=\varepsilon_{n} f\left(u_{n}\right)+\beta_{n} x_{n}+\left(1-\beta_{n}-\varepsilon_{n}\right) P_{C}\left(u_{n}-\lambda_{n} B u_{n}\right), \quad \forall n \geq 1,
\end{array}\right.
$$

where $\left\{\epsilon_{n}\right\},\left\{\beta_{n}\right\}$ are two sequences in $(0,1)$ and $\left\{r_{k, n}\right\}, k \in\{1,2,3, \ldots, M\}$ are a real sequence in $(0, \infty)$ satisfy the following conditions:

(C1) $\lim _{n \rightarrow \infty} \epsilon_{n}=0$ and $\sum_{n=1}^{\infty} \varepsilon_{n}=\infty$,

(C2) $0<\lim \inf _{n \rightarrow \infty} \beta_{n} \leq \lim \sup _{n \rightarrow \infty} \beta_{n}<1$,

(C3) $\left\{\alpha_{n}\right\},\left\{\lambda_{n}\right\} \subset[e, g] \subset(0,2 \xi), \lim _{n \rightarrow \infty} \alpha_{n}=0$ and $\lim _{n \rightarrow \infty} \lambda_{n}=0$,

(C4) $\left\{\delta_{n}\right\} \subset[0, b]$, for some $b \in(0,1)$ and $\lim _{n \rightarrow \infty}\left|\delta_{n+1}-\delta_{n}\right|=0$,

(C5) $\lim \inf _{n \rightarrow \infty} r_{k, n}>0$ and $\lim _{n \rightarrow \infty}\left|r_{k, n+1}-r_{k, n}\right|=0$ for each $k \in\{1,2,3, \ldots, M\}$,

Then, $\left\{x_{n}\right\}$ and $\left\{u_{n}\right\}$ converge strongly to a point $z \in \Theta$ which is the unique solution of the variational inequality

$$
\langle(f(z)-z, x-z\rangle \geq 0, \quad \forall x \in \Theta .
$$

Equivalently, we have $z=P_{\Theta} f(z)$.

Proof. Put $T_{n} \equiv I$ for all $n \geq 1$ and for all $x \in C$. Then $W_{n}=I, A=I$ and $\gamma=1$. The conclusion follows from Theorem 3.2. This completes the proof.

If $\delta_{n}=0$ and $M=1$, in Theorem 3.2, then we can obtain the following result immediately.

Corollary 3.4. Let $C$ be a nonempty closed convex subset of a real Hilbert space $H$, let $F_{k}, k \in\{1,2,3, \ldots, M\}$ be a bifunction from $C \times C$ to $\mathbb{R}$ satisfying (A1)-(A4), let $\left\{T_{n}\right\}$ be an infinite family of nonexpansive mappings of $C$ into itself and let $B$ be $\xi$-inverse strongly monotone such that

$$
\Theta:=\cap_{n=1}^{\infty} F\left(T_{n}\right) \cap E P(F) \cap \operatorname{VI}(C, B) \neq \emptyset .
$$

Let $f$ be a contraction of $H$ into itself with $\eta \in(0,1)$ and let $A$ be a strongly positive linear bounded operator on $H$ with coefficient $\bar{\gamma}>0$ and $0<\gamma<\frac{\bar{\gamma}}{\eta}$. Let $\left\{x_{n}\right\},\left\{y_{n}\right\}$ and $\left\{u_{n}\right\}$ be sequences generated by

$$
\left\{\begin{array}{l}
x_{1}=x \in C \text { chosen arbitrary, } \\
F\left(u_{n}, y\right)+\frac{1}{r_{n}}\left\langle y-u_{n}, u_{n}-x_{n}\right\rangle \geq 0, \quad \forall y \in C, \\
x_{n+1}=\varepsilon_{n} \gamma f\left(u_{n}\right)+\beta_{n} x_{n}+\left(\left(1-\beta_{n}\right) I-\varepsilon_{n} A\right) P_{C}\left(W_{n} u_{n}-\lambda_{n} B W_{n} u_{n}\right), \quad \forall n \geq 1,
\end{array}\right.
$$


where $\left\{W_{n}\right\}$ is the sequence generated by (3.1) and $\left\{\epsilon_{n}\right\},\left\{\beta_{n}\right\}$ are two sequences in $(0,1)$ and $\left\{r_{n}\right\}$ are a real sequence in $(0, \infty)$ satisfy the following conditions:

(C1) $\lim _{n \rightarrow \infty} \epsilon_{n}=0$ and $\sum_{n=1}^{\infty} \varepsilon_{n}=\infty$,

(C2) $0<\lim \inf _{n \rightarrow \infty} \beta_{n} \leq \lim \sup _{n \rightarrow \infty} \beta_{n}<1$,

(C3) $\left\{\lambda_{n}\right\} \subset[e, g] \subset(0,2 \xi)$ and $\lim _{n \rightarrow \infty} \lambda_{n}=0$,

(C4) $\lim \inf _{n \rightarrow \infty} r_{n}>0$ and $\lim _{n \rightarrow \infty}\left|r_{n+1}-r_{n}\right|=0$.

Then, $\left\{x_{n}\right\}$ and $\left\{u_{n}\right\}$ converge strongly to a point $z \in \Theta$ which is the unique solution of the variational inequality

$$
\langle(A-\gamma f) z, x-z\rangle \geq 0, \quad \forall x \in \Theta .
$$

Equivalently, we have $z=P_{\Theta}(I-A+\gamma f)(z)$.

\section{Acknowledgements}

The authors were supported by the Centre of Excellence in Mathematics, the Commission on Higher Education, Thailand. CJ was partially supported by Rajamangala University of Technology Rattanakosin Research and Development Institute, the Thailand Research Fund and the Commission on Higher Education under Grant No. MRG5480206. The authors thank the referees for their careful reading of the article and useful comments.

\section{Author details}

${ }^{1}$ Department of Mathematics, Faculty of Science, King Mongkut's University of Technology Thonburi, Bangkok 10140, Thailand ${ }^{2}$ Centre of Excellence in Mathematics, CHE, Si Ayuthaya Road, Bangkok 10400, Thailand ${ }^{3}$ Department of Mathematics, Faculty of Liberal Arts, Rajamangala University of Technology Rattanakosin, Nakhon Pathom 73170, Thailand

\section{Authors' contributions}

All authors contribute equally and significantly in this research work. All authors read and approved the final manuscript.

\section{Competing interests}

The authors declare that they have no competing interests.

Received: 29 November 2011 Accepted: 26 April 2012 Published: 26 April 2012

References

1. Blum, E, Oettli, W: From optimization and variational inequalities to equilibrium problems. Math Stud. 63, 123-145 (1994)

2. Agarwal, RP, Cho, YJ, Petrot, N: Systems of general nonlinear set-valued mixed variational inequalities problems in Hilbert spaces. Fixed Point Theory Appl 31 (2011). 2011

3. Combettes, PL, Hirstoaga, SA: Equilibrium programming in Hilbert spaces. J Nonlinear Convex Anal. 6, 29-41 (2005)

4. Cho, YJ, Petrot, N: On the system of nonlinear mixed implicit equilibrium problems in Hilbert spaces. J Inequal Appl 12 (2010). Article ID 437976, 2010

5. Cho, YJ, Petrot, N: An optimization problem related to generalized equilibrium and fixed point problems with applications. Fixed Point Theory. 11, 237-250 (2010)

6. Cho, YJ, Petrot, N: Regularization and iterative method for general variational inequality problem in Hilbert spaces. $J$ Inequal Appl 21 (2011). 2011

7. Cho, YJ, Argyros, IK, Petrot, N: Approximation methods for common solutions of generalized equilibrium, systems of nonlinear variational inequalities and fixed point problems. Comput Math Appl. 60, 2292-2301 (2010). doi:10.1016/j. camwa.2010.08.021

8. Cho, YJ, Kang, Jl, Qin, X: Convergence theorems based on hybrid methods for generalized equilibrium problems and fixed point problems. Nonlinear Anal. 71, 4203-4214 (2009). doi:10.1016/j.na.2009.02.106

9. Chen, JW, Cho, YJ, Kim, JK, Li, J: Multiobjective optimization problems with modified objective functions and cone constraints and applications. J Global Optim. 49, 137-147 (2011). doi:10.1007/s10898-010-9539-3

10. Colao, V, Marino, G, Xu, HK: An iterative method for finding common solutions of equilibrium and fixed point problems. J Math Anal Appl. 344, 340-352 (2008). doi:10.1016/j.jmaa.2008.02.041

11. Chantarangsi, W, Jaiboon, C, Kumam, P: A viscosity hybrid steepest descent method for generalized mixed equilibrium problems and variational inequalities, for relaxed cocoercive mapping in Hilbert spaces. Abst Appl Anal 39 (2010). Article ID 390972, 2010

12. He, H, Liu, S, Cho, YJ: An explicit method for systems of equilibrium problems and fixed points of infinite family of nonexpansive mappings. J Comput Appl Math. 235, 4128-4139 (2011). doi:10.1016/j.cam.2011.03.003

13. Jaiboon, C: The hybrid steepest descent method for addressing fixed point problems and system of equilibrium problems. Thai J Math. 8, 275-292 (2010) 
14. Moudafi, A, Thera, M: Proximal and dynamical approaches to equilibrium problems. Lecture Note in Economics and Mathematical Systems 477, 187-201 (1999). Springer-Verlag, New York. doi:10.1007/978-3-642-45780-7_12

15. Peng, JW, Yao, JC: A viscosity approximation scheme for system of equilibrium problems, nonexpansive mappings and monotone mappings. Nonlinear Anal. 71, 6001-6010 (2009). doi:10.1016/j.na.2009.05.028

16. Qin, X, Chang, SS, Cho, YJ: Iterative methods for generalized equilibrium problems and fixed point problems with applications. Nonlinear Anal Real World Appl. 11, 2963-2972 (2010). doi:10.1016/j.nonrwa.2009.10.017

17. Takahashi, S, Takahashi, W: Viscosity approximation methods for equilibrium problems and fixed point problems in Hilbert spaces. J Math Anal Appl. 331, 506-515 (2007). doi:10.1016/j.jmaa.2006.08.036

18. Yao, Y, Cho, YJ, Liou, Y: Iterative algorithms for variational inclusions, mixed equilibrium problems and fixed point problems approach to optimization problems. Central Eur J Math. 9, 640-656 (2011). doi:10.2478/s11533-011-0021-3

19. Yao, Y, Cho, YJ, Liou, Y: Algorithms of common solutions for variational inclusions, mixed equilibrium problems and fixed point problems. Eur J Oper. 212, 242-250 (2011). doi:10.1016/j.ejor.2011.01.042

20. Chang, SS, Joseph Lee, HW, Chan, CK: A new method for solving equilibrium problem fixed point problem and variational inequality problem with application to optimization. Nonlinear Anal. 70, 3307-3319 (2009). doi:10.1016/j. na.2008.04.035

21. Su, Y, Shang, M, Qin, X: An iterative method of solution for equilibrium and optimization problems. Nonlinear Anal. 69, 2709-2719 (2008). doi:10.1016/j.na.2007.08.045

22. Yao, Y, Liou, YC, Yao, YC: Convergence theorem for equilibrium problems and fixed point problems of infinite family of nonexpansive mappings. Fixed Point Theory Appl 12 (2007). Article ID 64363, 2007

23. Bauschke, HH, Borwein, JM: On projection algorithms for solving convex feasibility problems. SIAM Rev. 38, 367-426 (1996). doi:10.1137/S0036144593251710

24. Combettes, PL: The foundations of set theoretic estimation. Proceedings of the IEEE. 81, 182-208 (1993)

25. Goebeland, K, Kirk, WA: Topics in Metric Fixed Point Theory. Cambridge University Press, Cambridge, UK (1990)

26. Takahashi, W: Nonlinear Functional Analysis. Yokohama Publishers, Yokohama (2000)

27. Marino, G, Xu, HK: A general iterative method for nonexpansive mapping in Hilbert spaces. J Math Anal Appl. 318, 43-52 (2006). doi:10.1016/j.jmaa.2005.05.028

28. Takahashi, W, Toyoda, M: Weak convergence theorems for nonexpansive mappings and monotone mappings. J Optim Theory Appl. 118, 417-428 (2003). doi:10.1023/A:1025407607560

29. Yamada, I: The hybrid steepest descent method for the variational inequality problem of the intersection of fixed point sets of nonexpansive mappings. In: Butnariu D, Censor Y, Reich S (eds.) Inherently Parallel Algorithm for Feasibility and Optimization. pp. 473-504. Elsevier (2001)

30. Colao, V, Marino, G: Strong convergence for a minimization problem on point of equilibrium and common fixed points of family of nonexpansive mappings. Nonlinear Anal. 73, 3513-3524 (2010). doi:10.1016/..na.2010.07.011

31. Opial, Z: Weak convergence of the sequence of successive approximations for nonexpansive mappings. Bull Am Math Soc. 73, 595-597 (1967)

32. Suzuki, T: Strong convergence of krasnoselskii and mann's type sequences for one-parameter nonexpansive semigroups without bochner integrals. J Math Anal Appl. 305, 227-239 (2005). doi:10.1016/j.jmaa.2004.11.017

33. Xu, HK: Viscosity approximation methods for nonexpansive mappings. J Math Anal Appl. 298, 279-291 (2004). doi:10.1016/j.jmaa.2004.04.059

34. Shimoji, K, Takahashi, W: Strong convergence to common fixed points of infinite nonexpansive mappings and applications. Taiwan J Math. 5, 387-404 (2001)

35. Zhang, SS, Joseph Lee, JH, Chan, CK: Algorithms of common solutions for quasi-variational inclusion and fixed point problems. Appl Math Mech. 29(5):571-581 (2008). doi:10.1007/s10483-008-0502-y

doi:10.1186/1029-242X-2012-101

Cite this article as: Onjai-uea et al:: Convergence of iterative sequences for fixed points of an infinite family of nonexpansive mappings based on a hybrid steepest descent methods. Journal of Inequalities and Applications 2012 2012:101

\section{Submit your manuscript to a SpringerOpen ${ }^{\circ}$ journal and benefit from:}

- Convenient online submission

Rigorous peer review

- Immediate publication on acceptance

- Open access: articles freely available online

- High visibility within the field

- Retaining the copyright to your article

Submit your next manuscript at $\gg$ springeropen.com 